\title{
Optimal Portfolio Strategy with Discounted Stochastic Cash Inflows When the Stock Price Is a Semimartingale
}

\author{
Onthusitse Baraedi, Elias Offen \\ Department of Mathematics University of Botswana, Gaborone, Botswana \\ Email: goorabax@yahoo.com,offen@gmail.com
}

How to cite this paper: Baraedi, O. and Offen, E. (2016) Optimal Portfolio Strategy with Discounted Stochastic Cash Inflows When the Stock Price Is a Semimartingale. Journal of Mathematical Finance, 6, 660684.

http://dx.doi.org/10.4236/jmf.2016.64047

Received: August 19, 2016

Accepted: November 6, 2016

Published: November 9, 2016

Copyright $\odot 2016$ by authors and Scientific Research Publishing Inc. This work is licensed under the Creative Commons Attribution International License (CC BY 4.0).

http://creativecommons.org/licenses/by/4.0/

\begin{abstract}
This paper discusses optimal portfolio with discounted stochastic cash inflows (SCI). The cash inflows are invested into a market that is characterized by a stock and a cash account. It is assumed that the stock and the cash inflows are stochastic and the stock is modeled by a semi-martingale. The Inflation linked bond and the cash inflows are Geometric. The cash account is deterministic. We do some scientific analyses to see how the discounted stochastic cash inflow is affected by some of the parameters. Under this setting, we develop an optimal portfolio formula and later give some numerical results.
\end{abstract}

\section{Keywords}

Stochastic Cash Inflows, Portfolio, Inflation-Linked Bond, Semimartingale

\section{Introduction}

For example in financial mathematics, the classical model for a stock price is that of a geometric Brownian motion. However, it is argued that this model fails to capture properly the jumps in price changes. A more realistic model should take jumps into account. In the Jump diffusion model, the underlying asset price has jumps superimposed upon a geometric Brownian motion. The model therefore consists of a noise component generated by the Wiener process, and a jump component. It involves modelling option prices and finding the replicating portfolio. Researchers have increasingly been studying models from economics and from the natural sciences where the underlying randomness contains jumps. According to Nkeki [1], the wars, decisions of the Federal Reserve, other central banks, and other news can cause the 
stock price to make a sudden shift. To model this, one would like to represent the stock price by a process that has jumps (Bass [2]). Liu et al. (2003) [3] solved for the optimal portfolio in a model with stochastic volatility and jumps when the investor can trade the stock and a risk-free asset only. They also found that Liu and Pan (2003) [4] extended this paper to the case of a complete market. Arai [5] considered an incomplete financial market composed of $d$ risky assets and one riskless asset. Branger and Larsen [6] solved the portfolio planning problem of an ambiguity averse investor. They considered both an incomplete market where the investor can trade the stock and the bond only, and a complete market, where he also has access to derivatives. In Guo and $\mathrm{Xu}$ (2004) [7], researchers applied the mean-variance analysis approach to model the portfolio selection problem. They considered a financial market containing $d+1$ assets: $d$ risky stocks and one bond. The security returns are assumed to follow a jump-diffusion process. Uncertainty is introduced by Brown motion processes and Poisson processes The general method to solve mean-variance model is the dynamic programming. Dynamic programming technique was firstly introduced by Richard Bellman in the 1950s to deal with calculus of variations and optimal control prob-lems (Weber et al. [8]). Further developments have been obtained since then by a number of scholars including Florentin $(1961,1962)$ and Kushner (2006), among others. In Jin and Zhang [9], researchers solved the optimal dynamic portfolio choice problem in a jump-diffusion model with some realistic constraints on portfolio weights, such as the no-short-selling constraint and the no-borrowing constraint. Beginning with work of Nkeki [1] which involves optimization of the portfolio strategy using discounted stochastic cash inflows, this work explores optimal portfolio strategy using jump diffussion model.

In Nkeki [1], the stock price is modelled by continuous process which is geometric and but in this work we assume that the stock price process is driven by a semimartingale; defined in Shiryaev et al. [10]. The jump diffusion model combines the usual geometric Brownian motion for the diffusion and the general jump process such that the jump amplitudes are normally distributed.

Semimartingales as a tool of modelling stock prices processes has a number of advantages. For example this class contains discrete-time processes, diffusion processes, diffusion processes with jumps, point processes with independent increments and many other processes (Shiryaev [11]). The class of semimartingales is stable with respect to many transformations: absolutely continuous changes of measure, time changes, localization, changes of filtration and so on as stated in (Sharyaev [11]). Stochastic integration with respect to semimartingales describes the growth of capital in self-financing strategies. In this research, a sufficient maximum principle for the optimal control of jump diffusions is used showing dynamic programming and going applications to financial optimization problem in a market described by such process. For jump diffusions with jumps, a necessary maximum principle was given by Tang and $\mathrm{Li}$, see also Kabanov and Kohlmann ( $\varnothing \mathrm{ksendal}$ and Sulem [12]). If stochastic control satisfies the maximum principle conditions, then the control is indeed optimal 
for the stochastic control problem. It is believed that such results involves a useful complicated integro-differential equation (the Hamilton-Jacobi-Bellmann equation) in the jump diffusion case. The investor's stochastic Cash inflows (CSI) into the cash account, on inflation-linked bond and stock were considered. Most calculations and methods used were influenced by the works of Nkeki [1], Nkeki [13] Øksendal [14], $\varnothing$ ksendal and Sulem [12], Klebaner [15] and Cont and Tankov [16].

\section{Model Formulation}

Let $(\Omega, \mathcal{F}, \mathbb{P})$ be a probability space where $\mathcal{F}=\left(\mathcal{F}_{t}\right)_{t \geq 0}$ denotes the "flow of information" as discussed in the definition. Mathematically the latter means that $\mathcal{F}$ consists of $\sigma$-algebras, i.e. for all $s \leq t, \mathcal{F}_{s} \subset \mathcal{F}_{t} \subset \mathcal{F}$. The Brownian motions

$W(t)=\left(W^{I}(t), W^{S}(t)\right)$ is a 2 -dimensional process on a given filtered probability space $(\Omega, \mathcal{F}, F(\mathcal{F}), \mathbb{P}), t \in[0, T]$, where $\mathbb{P}$ is the real world probability measure, $t$ is the time period, $T$ is the terminal time period, $W^{I}(t)$ is the Brownian motion with respect to the "noise" arising from the inflation and $W^{I}(t)$ is the Brownian motion with respect to the "noise" arising from the stock market.

The dynamics of the cash account with the price $Q(t)$ is given by:

$$
\begin{gathered}
\mathrm{d} Q(t, I(t))=Q(t) r \mathrm{~d} t \\
Q(0)=1
\end{gathered}
$$

where $r$ is the short term interest as defined in Nkeki [1].

The price of the inflation-linked bond $B(t, I(t))$ is given by the dynamics:

$$
\begin{gathered}
\mathrm{d} B(t, I(t))=B(t, I(t))\left[\left(r+\sigma_{I} \phi_{I}\right) \mathrm{d} t+\sigma_{B} \cdot \mathrm{d} W(t)\right] \\
B(0, I(0))=b
\end{gathered}
$$

where $\sigma_{B}=\left(\sigma_{I}, 0\right)$ is the volatility of inflation-linked bond, $\phi_{I}$ is the market price of inflation risk, $I_{t}$ is the inflation index at time $t$ and has the dynamics:

$$
\mathrm{d} I(t)=I(t)\left[q \mathrm{~d} t+\sigma_{I} \cdot \mathrm{d} W(t)\right]
$$

where $q$ is the expected rate of inflation, which is the difference between nominal interest rate, $r$ real interest $\bar{r}$ and $\sigma_{I}$ is the volatility of inflation index.

Suppose the financial process ( stock return) $S=\left(S_{t}\right)_{t \geq 0}$ is given on a filtered probability space. Assume that $S(t)$ is of "exponential form".

$$
S_{t}=S_{0} \mathrm{e}^{H_{t}}, H_{0}=0, t \geq 0
$$

where $H=\left(H_{t}\right)_{t \geq 0}$ is a semi-martingale with respect to $\mathbb{F}$ and $\mathbb{P}$.

Using Itô formula for semimartingales (see Appendix) and then differentiating the process we have

$$
\mathrm{d} S_{t}=S_{t-} \mathrm{d} \hat{H}_{t}
$$

where

$$
\hat{H}_{t}=H_{t}+\frac{1}{2}\left\langle H^{c}\right\rangle_{s}+\sum_{0<s \leq t}\left(\mathrm{e}^{\Delta H_{s}}-1-\Delta H_{s}\right)
$$


Using random measure if jumps (see [11])

$$
\hat{H}_{t}=H_{t}+\frac{1}{2}\left\langle H^{c}\right\rangle_{s}+\left(\mathrm{e}^{x}-1-x\right) * \mu
$$

hence

$$
\mathrm{d} \hat{H}_{t}=\mathrm{d} \mathcal{B}(\varphi)_{t}+\mathrm{d} H_{t}^{c}+\frac{1}{2} \mathrm{~d}\left\langle H^{c}\right\rangle_{s}+\mathrm{d} \int_{0}^{t} \int_{|z| \leq 1}\left(\mathrm{e}^{z}-1\right) \mathrm{d}(\mu-v)+\mathrm{d} \int_{0}^{t} \int_{|z| \leq 1}\left(\mathrm{e}^{z}-1-\mathrm{z}\right) \mathrm{d} v
$$

Substituting on Equation (7) into Equation (50) we have

$$
\mathrm{d} S_{t}=S_{t-} \mathrm{d}\left(\mathcal{B}(\varphi)_{t}+H_{t}^{c}+\frac{1}{2}\left\langle H^{c}\right\rangle_{s}+\int_{0}^{t} \int_{|z| \leq 1}\left(\mathrm{e}^{z}-1\right) \mathrm{d}(\mu-v)+\int_{0}^{t} \int_{|z| \leq 1}\left(\mathrm{e}^{z}-1-z\right) \mathrm{d} v\right)
$$

We know that differential of our stock price can written as

$$
\mathrm{d} S(t)=S(t-)\left(\alpha \mathrm{d} t+\sigma_{1} \cdot \mathrm{d} W(t)+\text { jump }\right)
$$

where $\sigma_{1}=\left(\rho \sigma_{S}, \sqrt{1-\rho^{2}} \sigma_{S}\right)$ and $W(t)$ defined as before.

Now comparing Equation (8) with Equation (9), we can now see that when we equate the predictable parts we have

$$
\begin{gathered}
S_{t-}\left(\mathrm{d} \mathcal{B}(\varphi)_{t}+\frac{1}{2} \mathrm{~d}\left\langle H^{c}\right\rangle_{t}\right)=S_{t-} \alpha \mathrm{d} t \\
\mathrm{~d}\left(\mathcal{B}(\varphi)_{t}+\frac{1}{2}\left\langle H^{c}\right\rangle_{t}\right)=\alpha \mathrm{d} t, \mathcal{B}(\varphi)_{t}+\frac{1}{2}\left\langle H^{c}\right\rangle_{t}=\alpha t
\end{gathered}
$$

Equating the continuous parts we get

$$
\mathrm{d} H_{t}^{c}=\sigma_{1} \mathrm{~d} W_{t}, \quad H_{t}^{c}=\sigma_{1} W_{t}
$$

and the jump parts give

$$
\mathrm{d}\left(\int_{0}^{t} \int_{|z| \leq 1}\left(\mathrm{e}^{z}-1\right) \mathrm{d}(\mu-v)+\int_{0}^{t} \int_{|z| \leq 1}\left(\mathrm{e}^{z}-1-z\right) \mathrm{d} v\right)=\text { jump }
$$

and hence we let

$$
\int_{|z| \leq 1}\left(\mathrm{e}^{z}-1\right) \mathrm{d}(\mu-v)+\int_{|z| \leq 1}\left(\mathrm{e}^{z}-1-z\right) \mathrm{d} v=J
$$

From (11) it follows that $\left\langle H^{c}\right\rangle_{t}=\sigma_{1}^{2} t$ and hence it follows that

$$
\mathcal{B}(\varphi)_{t}=\alpha t-\frac{1}{2}\left\langle H^{c}\right\rangle_{t}=\left(\alpha-\frac{1}{2} \sigma_{1}^{2}\right) t
$$

Substituting Equation (12) into Equation (9) we have

$$
\mathrm{d} S(t)=S(t-)\left(\alpha \mathrm{d} t+\sigma_{1} \cdot \mathrm{d} W(t)+\int_{|z| \leq 1}\left(\mathrm{e}^{z}-1\right) \mathrm{d}(\mu-v)+\int_{|z| \leq 1}\left(\mathrm{e}^{z}-1-z\right) \mathrm{d} v\right)
$$

and further simply it to

$$
\mathrm{d} S(t)=S(t-)\left(\alpha \mathrm{d} t+\sigma_{1} \cdot \mathrm{d} W(t)+\int_{|z| \leq 1} \varphi_{1}(z) \mathrm{d}(\mu-v)+\int_{|z| \leq 1} \varphi_{2}(z) \mathrm{d} v\right)
$$

where $\varphi_{1}(z)=\varphi_{2}(z)+z=\mathrm{e}^{z}-1$

Using Itó's formula for jump diffusion 


$$
\begin{aligned}
S_{t}= & S_{0} \exp \left\{\left(\alpha-\frac{1}{2} \sigma_{1}^{2}\right) t+\sigma_{1} W_{t}+\int_{0}^{t} \int_{|z| \leq 0}\left(\ln \left(1-\varphi_{1}\right)+\varphi_{1}\right) v(\mathrm{~d} z, \mathrm{~d} s)\right. \\
& \left.+\int_{0}^{t} \int_{|z| \leq 0}\left(\ln \left(1-\varphi_{2}\right)+\varphi_{2}\right) v(\mathrm{~d} z, \mathrm{~d} s)+\int_{0}^{t} \int_{\mathbb{R}}\left(\ln \left(1-\varphi_{2}\right)\right)(\mu-v)(\mathrm{d} z, \mathrm{~d} s)\right\}
\end{aligned}
$$

(see Appendix). Hence we define the following

$$
\begin{gathered}
\Delta=\left(\begin{array}{c}
\sigma_{I} \phi_{I} \\
\alpha-r
\end{array}\right), \quad \Sigma=\left(\begin{array}{c}
\sigma_{B} \\
\sigma_{I}
\end{array}\right), \\
\theta=\left(\begin{array}{c}
\theta_{I} \\
\theta_{S}
\end{array}\right), \lambda=\left(\begin{array}{c}
\lambda \\
0
\end{array}\right)
\end{gathered}
$$

The market price of the market risk is given by

$$
\phi=\Sigma^{-1} \Delta=\left(\begin{array}{c}
\phi_{I} \\
\phi_{S}
\end{array}\right)=\left(\begin{array}{c}
\phi_{I} \\
\frac{\alpha-r-\rho \sigma_{1} \phi_{I}}{\sigma_{1} \sqrt{1-\rho^{2}}}
\end{array}\right)
$$

where, $\phi_{s}$ is the market price of stock market risk. We assume the process $P(t)$ which is geometric and with the no arbitrage conditions applied to it obtain the following stochastic differential equation,

$$
\mathrm{d} P(t)=P(t-)\left(-\phi_{t} \mathrm{~d} W_{t}+\int_{|z| \leq 1} \psi v(\mathrm{~d} z, \mathrm{~d} s)\right)
$$

Using Itó's formula for jump diffusion equation on 17 we have

$$
P(t)=P(0) \exp \left(-\phi W_{t}-\frac{1}{2} \phi^{2} t+\int_{0}^{t} \int_{|z| \leq 1} \psi_{1}(t, z) v(\mathrm{~d} z, \mathrm{~d} s)\right)
$$

where $\psi_{1}(t, z)=\ln (1+\psi(t, z))-\psi(t, z) \quad$ (see Appendix).

$P(t)$ is a martingale that is always positive and satisfies $\mathbb{E}[Z(T)]=1$.

Now we have the price density given by

$$
\Lambda(t)=\frac{P_{t}}{Q_{t}}
$$

where

$$
\Lambda=\frac{P(t)}{Q(t)}=P(0) \exp \left(-\phi W_{t}-\left(r+\frac{1}{2} \phi^{2}\right) t+\int_{0}^{t} \int_{|z| \leq 1} \psi_{1}(t, z) v(\mathrm{~d} z, \mathrm{~d} s)\right)
$$

\section{The Dynamics of Stochastic Cash Inflows}

The dynamics of the stochastic cash inflows with process, $D(t)$ is given by

$$
\mathrm{d} D(t)=D(t)\left(k d t+\sigma_{D}^{\prime} \mathrm{d} W(t)\right), D(0)=D_{0}
$$

where $\sigma_{D}=\left(\sigma_{1}^{D}, \sigma_{2}^{D}\right)^{\prime}$ is the volatility of the cash inflows and $\mathrm{k}$ is the expected growth rate of the cash inflows. $\sigma_{1}^{D}$ is the volatility arising from inflation and $\sigma_{2}^{D}$ is the volatility arising from the stock market.

Solving for $D(t)$ we use Itò's formula for continuous processes. Let $f(t)=\ln D_{t}$ and 


$$
\begin{gathered}
\mathrm{d} f=\frac{\partial f}{\partial t} \mathrm{~d} t+\frac{\partial f}{\partial D} \mathrm{~d} D(t)+\frac{1}{2} \frac{\partial^{2} f}{\partial D^{2}}[\mathrm{~d} D(t)]^{2} \\
=\frac{1}{D(t)}\left(D(t)\left(k \mathrm{~d} t+\sigma_{D}^{\prime} \mathrm{d} W(t)\right)\right)-\frac{1}{2} \frac{1}{D(t)^{2}}[D(t)]^{2} \sigma_{D}^{2} \mathrm{~d} t \\
=\left(k-\frac{1}{2} \sigma_{D}^{2}\right) \mathrm{d} t+\sigma_{D}^{\prime} \mathrm{d} W(t) \\
\mathrm{d} \ln D(t)=\left(k-\frac{1}{2} \sigma_{D}^{2}\right) \mathrm{d} t+\sigma_{D}^{\prime} \mathrm{d} W(t) \\
\ln D(t)-\ln D(0)=\int_{0}^{t}\left(k-\frac{1}{2} \sigma_{D}^{2}\right) \mathrm{d} s+\int_{0}^{t} \sigma_{D}^{\prime} \mathrm{d} W(s) \\
\ln \frac{D(t)}{D(0)}=\left(k-\frac{1}{2} \sigma_{D}^{2}\right) t+\sigma_{D}^{\prime} W(t) \\
D(t)=D(0) \exp \left(\left(k-\frac{1}{2} \sigma_{D}^{2}\right) t+\sigma_{D}^{\prime} W(t)\right)
\end{gathered}
$$

\section{The Dynamics of the Wealth Process}

If $X(t)$ is the wealth process and $\theta(t)=\left(\theta_{0}, \theta_{I}, \theta_{S}\right)$ is the admissible portfolio where $\theta_{0}$ is number of units in the cash account, $\theta_{I}$ is the number of units in the inflation bond and $\theta_{S}$ is the number of units in the stock. In an incomplete market with no arbitrage we have $\theta_{0}=1-\theta_{I}-\theta_{S}$. The dynamics of the wealth process is given by

$$
\begin{aligned}
\mathrm{d} X(t)= & \left(X_{t}(r+\theta \Delta)+D(t)\right) \mathrm{d} t+X_{t}(\Sigma \theta)^{\prime} \mathrm{d} W(t) \\
& +X_{t} \theta \lambda\left(\int_{|z| \leq 1} \varphi_{1}(\mu-v)(\mathrm{d} z, \mathrm{~d} s)+\int_{|z| \leq 1} \varphi_{2} v(\mathrm{~d} z, \mathrm{~d} s)\right)
\end{aligned}
$$

where

$$
\lambda=\exp \left(\alpha \delta t+\sigma_{1} \delta W_{t}+\int_{0}^{\delta t} \int_{|z| \leq 1} \varphi_{1}(\mu-v)(\mathrm{d} z, \mathrm{~d} s)+\int_{0}^{\delta t} \int_{|z| \leq 1} \varphi_{2} v(\mathrm{~d} z, \mathrm{~d} s)\right)
$$

(see Appendix). For $\mu=v=0$ we have the dynamics of the wealth process as

$$
\mathrm{d} X(t)=\left(X_{t}(r+\theta \Delta)+D(t)\right) \mathrm{d} t+X_{t}(\Sigma \theta)^{\prime} \mathrm{d} W(t)
$$

For the Poisson jump measure we have the dynamics of the wealth process as

$$
\begin{aligned}
\mathrm{d} X(t)= & \left(X_{t}(r+\theta \Delta)+D(t)\right) \mathrm{d} t+X_{t}(\Sigma \theta)^{\prime} \mathrm{d} W(t) \\
& +X_{t} \theta \lambda\left(\int_{|z| \leq 1} \varphi_{1}(\bar{N}-\bar{v})(\mathrm{d} z, \mathrm{~d} s)+\int_{|z| \leq 1} \varphi_{2} \bar{v}(\mathrm{~d} z, \mathrm{~d} s)\right)
\end{aligned}
$$

where $\bar{N}$ is the Poisson measure and $\bar{v}$ is the compensator on the Poisson measure $\bar{N}$.

\section{The Discounted Value of SCI}

In this Section, we introduce

Definition 1. The discounted value of the expected future SCI is defined as 


$$
\Psi(t)=\mathbb{E}_{t}\left(\int_{t}^{T} \frac{\Lambda(u)}{\Lambda(t)} D(u) \mathrm{d} u\right)
$$

where $\mathbb{E}_{t}=\mathbb{E}\left(\cdot \mid \mathrm{F}_{t}\right)$ is the conditional expectation with respect to the Brownian Filtration $\left\{\mathrm{F}_{t}\right\}_{t \geq 0}$ and $\Lambda(t)=P(t) \exp (-r t)$ is the stochastic discount factor which adjust for nominal interest rate and market price of risks for stock and inflation-linked bond (Nkeki [1]).

Proposition 1. If $\Psi(t)$ is the discounted value of the expected future SCI, then

$$
\Psi(t)=\frac{D(t)\left(\exp \left\{\left(k-r-\sigma_{D} \phi\right)(T-t)+\int_{0}^{T-t} \int_{|z| \leq 1} \psi_{1} v(\mathrm{~d} z, \mathrm{~d} s)\right\}-1\right)}{\eta+k-r-\sigma_{D} \phi}
$$

Proof. By definition 1, we have that

$$
\Psi(t)=D(t) \mathbb{E}_{t}\left(\int_{t}^{T} \frac{\Lambda(u) D(u)}{\Lambda(t) D(t)} \mathrm{d} u\right)
$$

Applying change of variable on 30 , we have

$$
\Psi(t)=D(t) \mathbb{E}_{t}\left(\int_{0}^{T-t} \frac{\Lambda(\tau) D(\tau)}{\Lambda(0) D(0)} \mathrm{d} \tau\right)
$$

starting with

$$
\begin{aligned}
\frac{\Lambda(\tau) D(\tau)}{\Lambda(0) D(0)}= & \exp \left\{-\phi W_{\tau}-\left(r+\frac{1}{2}\|\phi\|^{2}\right) \tau+\int_{0}^{T-t} \int_{|z| \leq 1} \psi_{1} v(\mathrm{~d} z, \mathrm{~d} s)\right\} \\
& \times \exp \left\{\left(k-\frac{1}{2}\left\|\sigma_{D}\right\|^{2}\right) \tau+\sigma_{D} W_{\tau}\right\} \\
= & \exp \left\{\left(\sigma_{D}-\phi\right) W_{\tau}+\left(k+r-\left(\frac{1}{2}\left\|\sigma_{D}\right\|^{2}+\frac{1}{2}\|\phi\|^{2}\right)\right) \tau+\int_{0}^{T-t} \int_{|z| \leq 1} \psi_{1} v(\mathrm{~d} z, \mathrm{~d} s)\right\} \\
= & \exp \left\{\left(\sigma_{D}-\phi\right) W_{\tau}+\left(k+r-\sigma_{D}^{\prime} \phi\right) \tau+\int_{0}^{T-t} \int_{|z| \leq 1} \psi_{1} v(\mathrm{~d} z, \mathrm{~d} s)\right\}
\end{aligned}
$$

we have

$$
\mathbb{E}\left[\frac{\Lambda(\tau) D(\tau)}{\Lambda(0) D(0)}\right]=\exp \left\{\left(k+r-\sigma_{D}^{\prime} \phi\right) \tau+\int_{0}^{T-t} \int_{|z| \leq 1} \psi_{1} v(\mathrm{~d} z, \mathrm{~d} s)\right\}
$$

and lastly

$$
\Psi(t)=D(t) \int_{0}^{T-t} \exp \left\{\left(k+r-\sigma_{D}^{\prime} \phi\right) \mathrm{d} \tau+\int_{0}^{T-t} \int_{|z| \leq 1} \psi_{1} v(\mathrm{~d} z, \mathrm{~d} s)\right\}
$$

We further take note that for $v=0$ we have the discounted value of the SCI as

$$
\Psi(t)=\frac{D(t)\left(\exp \left\{\left(k-r-\sigma_{D} \phi\right)(T-t)-1\right\}\right)}{\eta+k-r-\sigma_{D} \phi}
$$

The differential form of $\Psi(t)$ is given by

$$
\mathrm{d} \Psi(t)=\Psi(t)\left(\left(r+\sigma_{D} \phi\right) \mathrm{d} t+\sigma_{D}^{\prime} \mathrm{d} W(t)+\int_{|z| \leq 1} \psi_{1}(\mathrm{~d} z) \mathrm{d} t\right)-D(t) \mathrm{d} t
$$

Equation (32) is obtained by differentiating $\Psi(t)$ as shown in the proof below 


$$
\Psi(t)=\frac{D(t)\left(\exp \left\{\left(k-r-\sigma_{D}^{\prime} \phi\right)(T-t)+\int_{0}^{T-t} \int_{|z|<1} \psi_{1} v(\mathrm{~d} z, \mathrm{~d} s)\right\}-1\right)}{\eta+k-r-\sigma_{D}^{\prime} \phi}
$$

differentiating both sides,

$$
\begin{aligned}
\mathrm{d} \Psi(t)= & \frac{D(t) \mathrm{d}\left(\exp \left\{\beta(T-t)+\int_{0}^{T-t} \int_{|z| \leq 1} \psi_{1} v(\mathrm{~d} z, \mathrm{~d} s)\right\}-1\right)}{\eta+\beta} \\
& +\frac{\mathrm{d} D(t)\left(\exp \left\{\beta(T-t)+\int_{0}^{T-t} \int_{|z| \leq 1} \psi_{1} v(\mathrm{~d} z, \mathrm{~d} s)\right\}-1\right)}{\eta+\beta} \\
= & \frac{-(\eta+\beta) D(t)\left(\exp \left\{\beta(T-t)+\int_{0}^{T-t} \int_{|z| \leq 1} \psi_{1} v(\mathrm{~d} z, \mathrm{~d} s)\right\}\right)}{\eta+\beta} \mathrm{d} t \\
= & \frac{-(\eta+\beta) D(t)\left(\exp \left\{\beta(T-t)+\int_{0}^{T-t} \int_{|z| \leq 1} \psi_{1} v(\mathrm{~d} z, \mathrm{~d} s)-1\right\}\right)}{\eta+\beta} \mathrm{d} t-\frac{\beta+\eta}{\beta+\eta} D(t) \mathrm{d} t \\
& +\frac{D(t)\left(k \mathrm{~d} t+\sigma_{D}^{\prime} \mathrm{d} W(t)\right) \times\left(\exp \left\{\beta(T-t)+\int_{0}^{T-t} \int_{|z| \leq 1} \psi_{1} v(\mathrm{~d} z, \mathrm{~d} s)\right\}-1\right)}{\eta\left(k \mathrm{~d} t+\sigma_{D}^{\prime} \mathrm{d} W(t)\right) \times\left(\exp \left\{\beta(T-t)+\int_{0}^{T-t} \int_{|z| \leq 1} \psi_{1} v(\mathrm{~d} z, \mathrm{~d} s)\right\}-1\right)} \\
= & \Psi(t)\left(\left(r+\sigma_{D} \phi\right) \mathrm{d} t+\sigma_{D}^{\prime} \mathrm{d} W(t)+\int_{|z| \leq 1} \psi_{1} v(\mathrm{~d} z) \mathrm{d} t\right)-D(t) \mathrm{d} t \\
= & \frac{D(t)\left(\exp \left\{\beta(T-t)+\int_{0}^{T-t} \int_{|z| \leq 1} \psi_{1} v(\mathrm{~d} z, \mathrm{~d} s)-1\right\}\right)}{\eta+\beta} \\
& \times\left(-(\eta+\beta) \mathrm{d} t+k \mathrm{~d} t+\sigma_{D}^{\prime} \mathrm{d} W(t)\right)-D(t) \mathrm{d} t
\end{aligned}
$$

The current discounted cash inflows can be obtained by putting $t=0$ into Equation (28),

$$
\Psi(0)=\frac{D(0)\left(\exp \left\{\left(k-r-\sigma_{D} \phi\right)(T)+\int_{0}^{T} \int_{|z| \leq 1} \psi_{1} v(\mathrm{~d} z, \mathrm{~d} t)\right\}-1\right)}{\eta+k-r-\sigma_{D} \phi}
$$

If $r+\sigma_{D} \phi>k$ and $\int_{0}^{\infty} \int_{|z| \leq 1} \psi_{1} v(\mathrm{~d} z, \mathrm{~d} s)<\infty$ we can change the horizon by allowing our $\mathrm{T}$ to go up to $\infty$ i.e.

$$
\begin{aligned}
\lim _{T \rightarrow \infty} \Psi(0) & =\lim _{T \rightarrow \infty} \frac{D(0)\left(\exp \left\{\left(k-r-\sigma_{D} \phi\right)(T)+\int_{0}^{T} \int_{|z| \leq 1} \psi_{1} v(\mathrm{~d} z, \mathrm{~d} t)\right\}-1\right)}{\eta+k-r-\sigma_{D} \phi} \\
& =\frac{D(0)}{\eta+k-r-\sigma_{D} \phi}
\end{aligned}
$$

In case of deterministic case, we have $\phi=0$ and $\psi=0$, so 


$$
\Psi(0)=\frac{D(0)(\exp \{(k-r)(T)\}-1)}{k-r}
$$

and for $r>k$, and $T \rightarrow \infty$ we have

$$
\lim _{T \rightarrow \infty} \Psi(0)=\lim _{T \rightarrow \infty} \frac{D(0)(\exp \{(k-r)(T)\}-1)}{k-r}=\frac{D(0)}{r-k}
$$

Since $\Psi(0)=\Psi_{0}\left(T, D_{0}, k, r, \sigma_{D}\right)$ is a constant, if we are interested to see how it behaves with respect to $T, D_{0}, k, r$ and $\sigma_{D}$ we need to take $\Psi(0)$ as a function of $T, D_{0}, k, r$ and $\sigma_{D}$. Then we can look at the sensitivity analysis of $\Psi(0)$,

Finding partial derivatives of $\Psi_{0}$ we obtain the followng

Differentiating $\Psi_{0}$ with respect to $T$, we have

$$
\frac{\partial}{\partial T} \Psi_{0}=D_{0} \exp \left(\beta T+\int_{|z| \leq 1} \psi_{1} v(\mathrm{~d} \xi)\right)
$$

Differentiating $\Psi_{0}$ with respect to $D_{0}$, we have

$$
\frac{\partial}{\partial D_{0}} \Psi_{0}=\frac{1}{D_{0}} \Psi_{0}
$$

Differentiating $\Psi_{0}$ with respect to $k$, we have

$$
\frac{\partial}{\partial k} \Psi_{0}=\frac{1}{(\eta+\beta)^{2}}\left(((\eta+\beta) T-1)\left(\Psi_{0}+\frac{D_{0}}{\eta+\beta}\right)+D_{0}\right)
$$

Differentiating $\Psi_{0}$ with respect to $r$, we have

$$
\frac{\partial}{\partial r} \Psi_{0}=\frac{1}{(\eta+\beta)^{2}}\left((1-(\eta+\beta) T)\left(\Psi_{0}+\frac{D_{0}}{\eta+\beta}\right)-D_{0}\right)
$$

Differentiating $\Psi_{0}$ with respect to $\sigma_{D}$, we have

$$
\frac{\partial}{\partial \sigma_{D}} \Psi_{0}=\frac{1}{(\eta+\beta)^{2}}\left((1-(\eta+\beta) T)\left(\Psi_{0}+\frac{D_{0}}{\eta+\beta}\right)-D_{0}\right)
$$

where $\beta=k-r-\sigma_{D} \phi$ and $\eta=\int_{|z| \leq 1} \psi_{1} v(\mathrm{dz})$ and $\psi_{1}=\ln (1+\psi)-\psi$

The following calculations shows how we differentiated $\Psi(0)$ with respect to $T$

$$
\begin{aligned}
\Psi_{0} & =\frac{D(0)}{\beta+\eta}\left(\exp \left(\beta T+\int_{0}^{T} \int_{|z| \leq 1} \psi_{1} v(\mathrm{~d} z, \mathrm{~d} t)\right)-1\right) \\
& =\frac{D(0)}{\beta+\eta} \exp \left(\beta T+\int_{0}^{T} \int_{|z| \leq 1} \psi_{1} v(\mathrm{~d} z, \mathrm{~d} t)\right)-\frac{D_{0}}{\beta+\eta}
\end{aligned}
$$

differentiating with respect to $T$

$$
\begin{aligned}
\frac{\mathrm{d}}{\mathrm{d} T} \Psi_{0} & =\frac{D_{0}}{\beta+\eta} \exp \left(\beta T+\int_{0}^{T} \int_{|z| \leq 1} \psi_{1} v(\mathrm{~d} z, \mathrm{~d} t)\right) \times(\beta+\eta) \\
& =D_{0} \exp \left(\beta T+\int_{0}^{T} \int_{|z| \leq 1} \psi_{1} v(\mathrm{~d} z, \mathrm{~d} t)\right)
\end{aligned}
$$

We repeated the following procedure for all other variables.

When we have a deterministic case, differentiating $\Psi_{0}$ partially we have the fol- 
lowing

Differentiating $\Psi_{0}$ with respect to $T$, we have

$$
\frac{\partial}{\partial T} \Psi_{0}=D_{0} \exp (\beta T)
$$

Differentiating $\Psi_{0}$ with respect to $D_{0}$, we have

$$
\frac{\partial}{\partial D_{0}} \Psi_{0}=\frac{1}{\beta}\left(\frac{1}{D_{0}} \frac{\partial \Psi_{0}}{\partial T}-1\right)
$$

Differentiating $\Psi_{0}$ with respect to $r$, we have

$$
\frac{\partial}{\partial r} \Psi_{0}=\frac{1}{\beta^{2}}\left((1-\beta T) \frac{\partial \Psi_{0}}{\partial T}-D_{0}\right)
$$

Differentiating $\Psi_{0}$ with respect to $\sigma_{D}$, we have

$$
\frac{\partial}{\partial \sigma_{D}} \Psi_{0}=\frac{\phi}{\beta^{2}}\left((1-\beta T) \frac{\partial \Psi_{0}}{\partial T}-D_{0}\right)
$$

Differentiating $\Psi_{0}$ with respect to $k$, we have

$$
\frac{\partial}{\partial k}\left(\Psi_{0}\right)=\frac{1}{\beta^{2}}\left((\beta T-1) \frac{\partial \Psi_{0}}{\partial T}+D_{0}\right)
$$

Table 1 shows the sensitivity of variables. Sensitivity analysis can be incorporated into discounted cash inflows analysis by examining how the discounted cash inflows of each project changes with changes in the inputs used. These could include changes in revenue assumptions, cost assumptions, tax rate assumptions, and discount rates. It also enables management to have contingency plans in place if assumptions are not met. It also shows that the return on the project is sensitive to changes in the projected revenues and costs. Looking at Table 1 , one can see that changing a variable can make

Table 1. Simulation of the sensitivity analysis.

\begin{tabular}{ccccccccc}
\hline$T$ & $\frac{\partial \Psi_{0}}{\partial D_{0}}$ & $\frac{\partial \Psi_{0}}{\partial \sigma_{1}^{D}}$ & $\frac{\partial \Psi_{0}}{\partial \sigma_{2}^{D}}$ & $\frac{\partial \Psi_{0}}{\partial T}$ & $\frac{\partial \Psi_{0}}{\partial r}$ & $\frac{\partial \Psi_{0}}{\partial \theta_{I}}$ & $\frac{\partial \Psi_{0}}{\partial \theta_{S}}$ & $\frac{\partial \Psi_{0}}{\partial k}$ \\
\hline 1 & 1.00 & -4.01 & -4.83 & 100.4359 & -50.15 & -12.54 & -18.05 & 50.15 \\
2 & 2.01 & -16.09 & -19.36 & 100.87 & -201.16 & -50.29 & -72.42 & 201.16 \\
3 & 3.02 & -36.31 & -43.69 & 101.31 & -453.93 & -113.42 & -163.42 & 453.93 \\
4 & 4.04 & -64.753 & -77.90 & 101.76 & -809.34 & -202.34 & -291.36 & 809.34 \\
5 & 5.05 & -101.46 & -122.07 & 102.20 & -1268.27 & -317.07 & -456.58 & 1268.27 \\
6 & 6.08 & -146.53 & -176.29 & 102.64 & -1831.63 & -457.91 & -659.39 & 1831.63 \\
7 & 7.11 & -200.02 & -240.65 & 103.09 & -2500.31 & -625.08 & -900.11 & 2500.31 \\
8 & 8.14 & -262.02 & -315.24 & 103.54 & -3275.22 & -818.80 & -1179.08 & 3275.22 \\
9 & 9.18 & -332.58 & -400.14 & 103.99 & -4157.27 & -1039.32 & -1496.62 & 4157.27 \\
10 & 10.22 & -411.79 & -495.44 & 104.45 & -5147.39 & -1286.85 & -1853.06 & 5147.39 \\
\hline
\end{tabular}


an impact on the SCI. An investor must do the sensitivity analysis in order to know changes can be made on the market to improve the results of an investment.

\section{The Dynamics of the Value Process}

Proposition 2. If $V(t)$ is the value process and $V(t)=\Psi(t)+X(t)$ where $\Psi$ is the discounted value of the expected future SCI then the differential form of $V(t)$ is given by

$$
\begin{aligned}
\mathrm{d} V(t)= & \left(r\left(X_{t}+\Psi_{t}\right)+\Delta \theta X_{t}+\sigma_{D} \phi \Psi_{t}\right) \mathrm{d} t+\left(\sum \theta X_{t}+\sigma_{D} \Psi_{t}\right) \mathrm{d} W(t) \\
& +\Psi(t-) \int_{|z| \leq 1} \psi_{1} v(\mathrm{~d} z) \mathrm{d} t+\lambda \theta X(t-) \int_{|z| \leq 1} \varphi_{2} v(\mathrm{~d} z) \mathrm{d} t \\
& +\lambda \theta X(t-) \int_{|z| \leq 1} \varphi_{1}(\mu-v)(\mathrm{d} z) \mathrm{d} t
\end{aligned}
$$

Proof. Differentiating $V(t)$ and substituting Equations (32) and (26) on the differential obtained we have

$$
\begin{aligned}
\mathrm{d} V(t)= & \mathrm{d} \Psi(t)+\mathrm{d} X(t) \\
= & \Psi(t)\left(\left(r+\sigma_{D} \phi\right) \mathrm{d} t+\sigma_{D}^{\prime} \mathrm{d} W(t)\right)+\Psi(t-) \int_{|z| \leq 1} \psi_{1} v(\mathrm{~d} z) \mathrm{d} t-D(t) \mathrm{d} t \\
& +X(t)\left((r+\Sigma \theta) \mathrm{d} t+(\Sigma \theta)^{\prime} \mathrm{d} W(t)\right) \mathrm{d} t+\lambda \theta X(t-) \int_{|z| \leq 1} \varphi_{2} v(\mathrm{~d} z) \mathrm{d} t \\
& +\lambda \theta X(t-) \int_{|z| \leq 1} \psi_{2}(\mu-v)(\mathrm{d} z) \mathrm{d} t+D(t) \mathrm{d} t \\
= & \left(r(X(t)+\Psi(t))+\left(\sigma_{D} \phi \Psi(t)+\Delta \theta X(t)\right)\right) \mathrm{d} t+\left(\Psi(t) \sigma_{D}+X(t) \Sigma \theta\right)^{\prime} \mathrm{d} W(t) \\
& +\Psi(t-) \int_{|z| \leq 1} \psi_{1} v(\mathrm{~d} z) \mathrm{d} t+\lambda \theta X(t-) \int_{|z| \leq 1} \varphi_{2} v(\mathrm{~d} z) \mathrm{d} t \\
& +\lambda \theta X(t-) \int_{|z| \leq 1} \psi_{2}(\mu-v)(\mathrm{d} z) \mathrm{d} t
\end{aligned}
$$

For $\mu=v=0$, the jump part becomes zero and we obtain

$$
\mathrm{d} V(t)=\left(r\left(X_{t}+\Psi_{t}\right)+\Delta \theta X_{t}+\sigma_{D} \phi \Psi_{t}\right) \mathrm{d} t+\left(\Sigma \theta X_{t}+\sigma_{D} \Psi_{t}\right) \mathrm{d} W(t)
$$

\section{Finding 0ptimal Portfolio}

Theorem 3. Let $X(t)$ be the worth process whose dynamics is defined by Equation (23), $\Psi(t)$ the discounted value of expected future stochastic cash inflow as defined in proportion (1), $V(t)$ the value process as defined in proportion (2) and $U(v)=\frac{v^{1-\gamma}}{1-\gamma}$ the utility function and if we assume that $v=0$, the optimal portfolio is given by $\theta(t)^{*}=\left(\theta_{0}^{*}, \theta_{I}^{*}, \theta_{S}^{*}\right)$ where

$$
\left(\begin{array}{c}
\theta_{I}^{*} \\
\theta_{S}^{*}
\end{array}\right)=\left(\begin{array}{c}
\frac{\phi_{I} V^{*}(t)}{\sigma_{I} \gamma X^{*}(t)}-\frac{\sigma_{1}^{D} \Psi(t)}{\sigma_{I} X^{*}(t)} \\
\frac{\left(\sigma_{I}(\alpha-r)+\sigma_{s} \phi_{I} \rho\left(\sigma_{s} \sqrt{1-\rho^{2}}-\sigma_{I}\right)\right) V^{*}(t)}{\sigma_{s}^{2} \sigma_{I}\left(1-\rho^{2}\right) \gamma X^{*}(t)}-\frac{\left(\sigma_{I} \sigma_{2}^{D}-\sigma_{s} \sigma_{1}^{D}\right) \Psi(t)}{\sigma_{s} \sigma_{I} \sqrt{1-\rho^{2}} X^{*}(t)}
\end{array}\right)
$$

and 


$$
\begin{aligned}
\theta_{0}^{*}= & 1-\theta_{I}^{*}-\theta_{s}^{*} \\
= & 1-\frac{\phi_{I} V^{*}(t)}{\sigma_{I} \gamma X^{*}(t)}+\frac{\sigma_{1}^{D} \Psi(t)}{\sigma_{I} X^{*}(t)}-\frac{\left(\sigma_{I}(\alpha-r)+\sigma_{s} \phi_{I} \rho\left(\sigma_{s} \sqrt{1-\rho^{2}}-\sigma_{I}\right)\right) V^{*}(t)}{\sigma_{s}^{2} \sigma_{I}\left(1-\rho^{2}\right) \gamma X^{*}(t)} \\
& +\frac{\left(\sigma_{I} \sigma_{2}^{D}-\sigma_{s} \sigma_{1}^{D}\right) \Psi(t)}{\sigma_{s} \sigma_{I} \sqrt{1-\rho^{2}} X^{*}(t)}
\end{aligned}
$$

The proof is given in Appendix.

From Equation (71), $\frac{\left(\sigma_{I}(\alpha-r)+\sigma_{s} \phi_{I} \rho\left(\sigma_{s} \sqrt{1-\rho^{2}}-\sigma_{I}\right)\right) V^{*}(t)}{\sigma_{s}^{2} \sigma_{I}\left(1-\rho^{2}\right) \gamma X^{*}(t)}$ represents the classical portfolio strategy at time $t$ and $\frac{\left(\sigma_{I} \sigma_{2}^{D}-\sigma_{s} \sigma_{1}^{D}\right) \Psi(t)}{\sigma_{s} \sigma_{I} \sqrt{1-\rho^{2}} X^{*}(t)}$ represents the intertemporal hedging term that offset shock from the SCI at time $t$.

\section{Some Numerical Values}

Figure 1 was obtained by setting $T=10, \phi_{I}=0.08, r=0.04, k=0.099, \alpha=0.09$, $\sigma_{d}=[0.25 ; 0.36], \sigma_{s}=0.4, \sigma_{I}=0.4, D=100, \rho=0.6$ and $\gamma=0.5$ in Equation (70). This figure shows that when $t=0$, the portfolio value is 0.151 which is equivalent to $15.1 \%$ when the value of the wealth is 40,000 and the portfolio value is 0.159 which is equivalent to $15.9 \%$ when the value of the wealth is $1,000,000$. When $t=10$, the portfolio value is 0.16 which is equivalent to $16 \%$ when the value of the wealth is 40,000

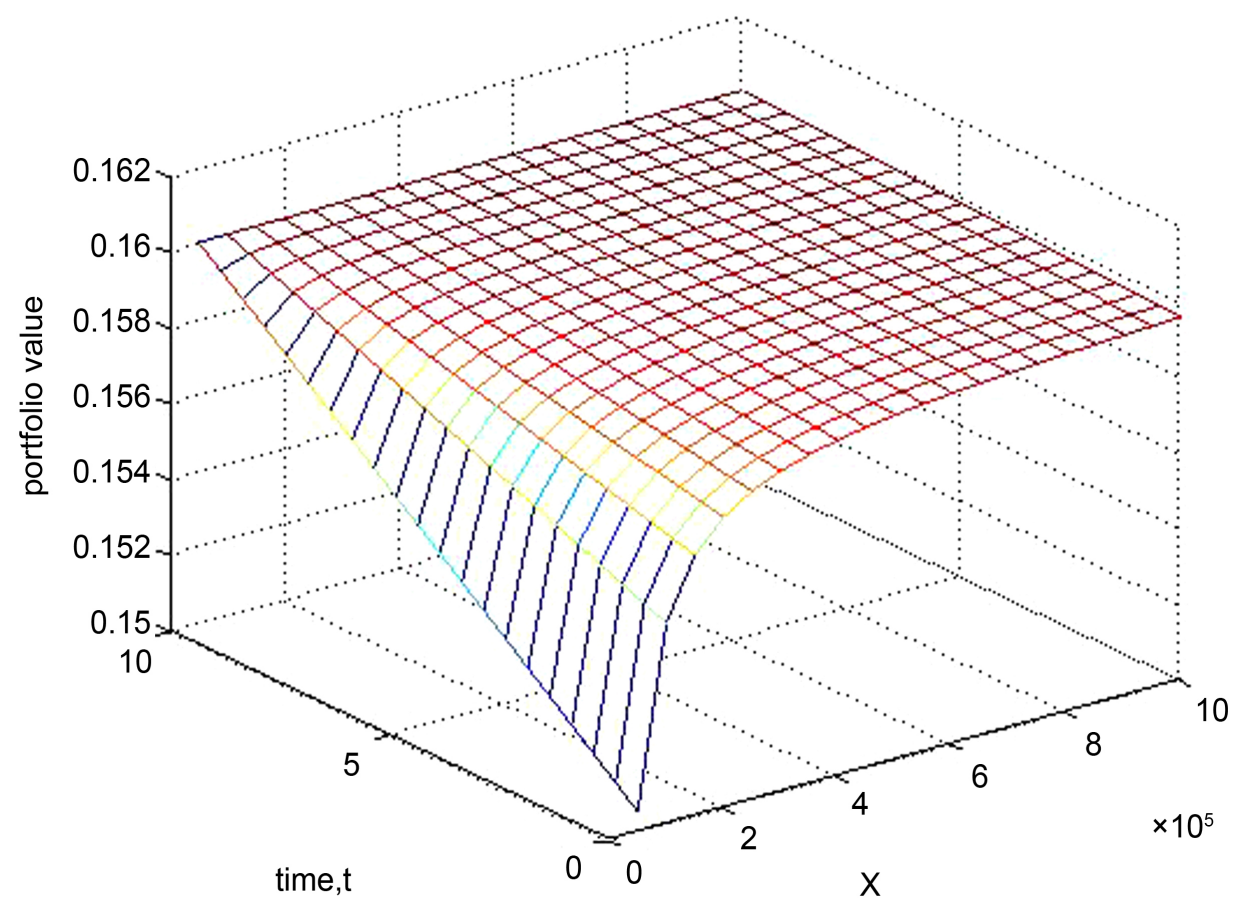

Figure 1. Portfolio value in inflation-linked bond. 
and the portfolio value is 0.1604 which is equivalent to $16.04 \%$ when the value of the wealth is $1,000,000$. This shows that there is a huge increase on the portfolio value from $t=0$ to $t=10$ when the value of the wealth is small and there in less change when the value of the wealth is large.

Figure 2 was obtained by setting $T=10, \phi_{I}=0.08, r=0.04, k=0.099, \alpha=0.09$, $\sigma_{d}=[0.25 ; 0.36], \quad \sigma_{s}=0.4, \sigma_{I}=0.4, D=100, \rho=0.6$ and $\gamma=0.5$ in Equation (71). This figure shows that when $t=0$, the portfolio value is 0.907 which is equivalent to $90.7 \%$ when the value of the wealth is 40,000 and the portfolio value is 0.9019 which is equivalent to $90.19 \%$ when the value of the wealth is $1,000,000$. When $t=10$, the portfolio value is 0.9017 which is equivalent to $90.17 \%$ when the value of the wealth is 40,000 and the portfolio value is 0.9017 which is equivalent to $90.17 \%$ when the value of the wealth is $1,000,000$. This shows that there is a huge decrease on the portfolio value from $t=0$ to $t=10$ when the value of the wealth is small and there in less change when the value of the wealth is large.

Figure 3 was obtained by setting $T=10, \phi_{I}=0.08, r=0.04, k=0.099, \alpha=0.09$, $\sigma_{d}=[0.25 ; 0.36], \quad \sigma_{s}=0.4, \sigma_{I}=0.4, D=100, \rho=0.6$ and $\gamma=0.5$ in Equation (72). This figure shows that when $t=0$, the portfolio value is -0.057 which is equivalent to $-5.7 \%$ when the value of the wealth is 40,000 and the portfolio value is -0.0613 which is equivalent to $-6.13 \%$ when the value of the wealth is $1,000,000$. When $t=10$, the portfolio value is -0.0615 which is equivalent to $-6.15 \%$ when the value of the wealth is 40,000 and the portfolio value is -0.0613 which is equivalent to $6.13 \%$ when the value of the wealth is $1,000,000$. This shows that there is a huge decrease on the portfolio value from $t=0$ to $t=10$ when the value of the wealth is small and

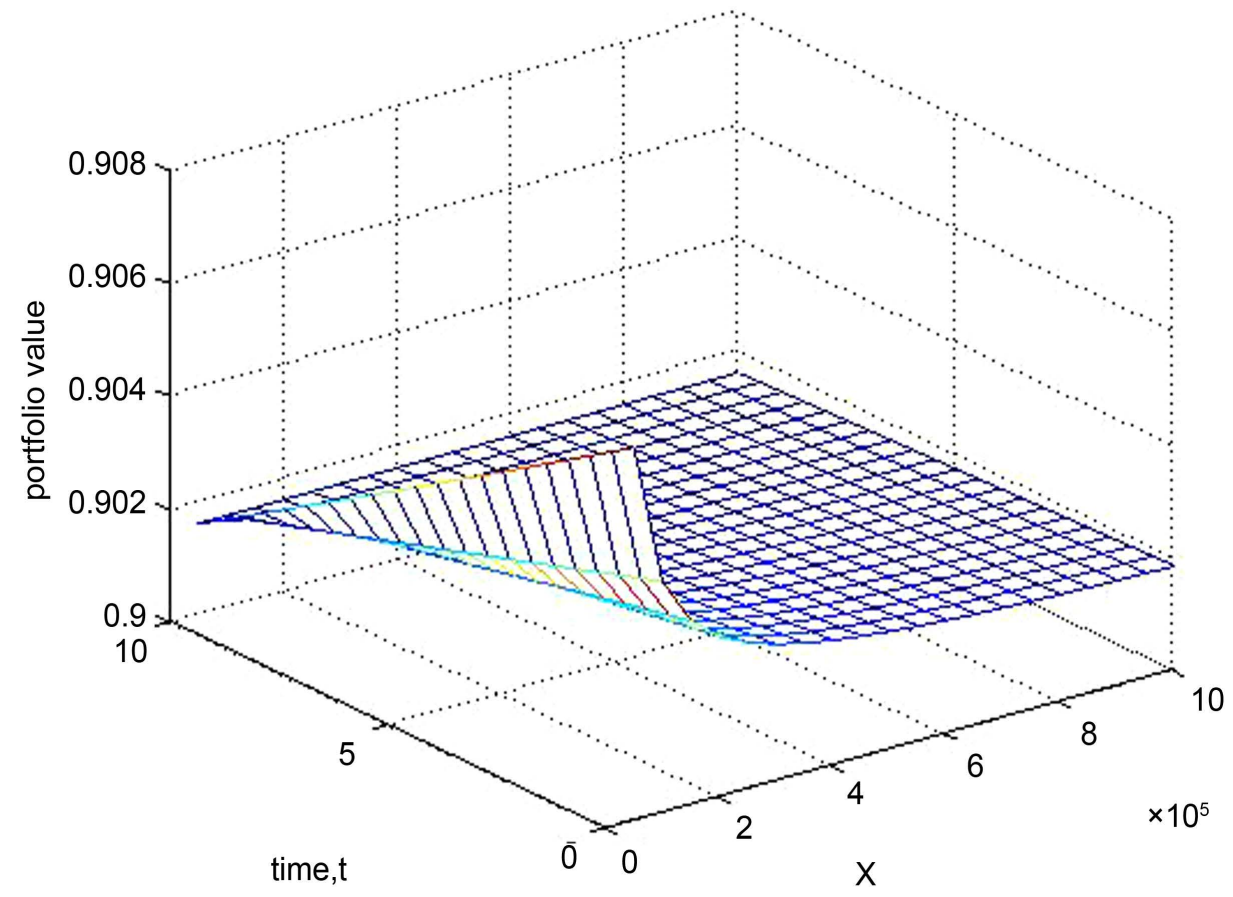

Figure 2. Portfolio value in stock. 


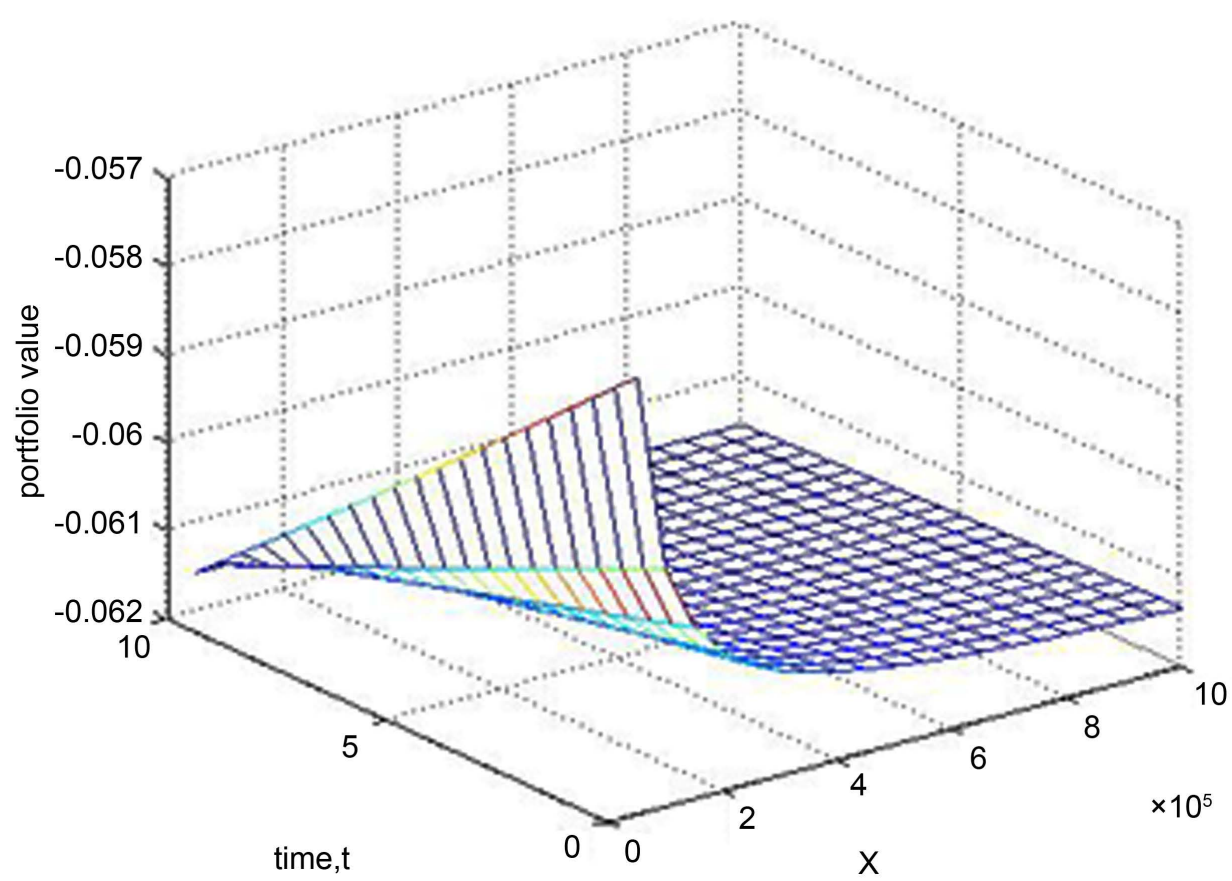

Figure 3. Portfolio value in cash account.

there in less change when the value of the wealth is large.

For $v \neq 0$, we have a problem because we cannot solve the equation explicitly. we need to come up with a computer program.

\section{Conclusion}

Semimartingales seems to model financial processes better since the cater for the jumps that occur in the system. The continuous processes may be convenient because one can easily produce results. For example, in our situation we managed to find the portfolio for continuous processes but we couldn't for the ones with jumps. This work can be extended designing a MATLAB program that will solve the equation for portfolio $\theta$.

\section{Acknowledgements}

We thank the editor and the referee for their comments. We also thank Professor E. Lungu for the guidance he gave us on achieving this. Lastly, we thank the University of Botswana for the resources we used to come up with this paper. Not forgetting the almighty God, the creator.

\section{References}

[1] Nkeki, C.I. (2013) Optimal Portfolio Strategy with Discounted Stochastic Cash Inflows, Journal of Mathematical Finance, 3, 130-137. http://dx.doi.org/10.4236/jmf.2013.31012

[2] Bass, B.F. (2004) Stochastic Differential Equations with Jumps. Probability Surveys, 1, 1-19. http://dx.doi.org/10.1214/154957804100000015

[3] Liu, J., Pan, J. and Wang, T. (2001) An Equilibrium Model of Rare Event Premia. Working 
Paper, UCLA, MIT Sloan, and UBC.

[4] Liu, J. and Pan, J. (2003) Dynamic Derivative Strategies. Journal of Financial Economics, 69, 401-430. http://dx.doi.org/10.1016/S0304-405X(03)00118-1

[5] Arai, T. (2004) Minimal Martingale Measures for Jump Diffussion Processes. Journal of Applied Probability, 41, 263-270. http://dx.doi.org/10.1017/S0021900200014194

[6] Branger, N. and Larsen, L.S. (2013) Robust Portfolio Choice with Uncertainty about Jump and Diffusion Risk. Journal of Banking and Finance, 37, 5036-5047.

http://dx.doi.org/10.1016/j.jbankfin.2013.08.023

[7] Guo, W. and Xu, C. (2004) Optimal Portfolio Selection When Stock Prices Follow an JumpDiffusion Process. Mathematical Methods of Operations Research, 60, 485-496. http://dx.doi.org/10.1007/s001860400365

[8] Azevedo, N., Pinheiro, D. and Weber, G.W. (2014) Dynamic Programming for a MarkovSwitching Jump Diffusion. Journal of Computational and Applied Mathematics, 267, 1-19. http://dx.doi.org/10.1016/j.cam.2014.01.021

[9] Jin, X. and Zhang, K. (2013) Dynamic Optimal Portfolio Choice in a Jump-Diffusion Model with Investment Constraints. Journal of Banking and Finance, 37, 1733-1746. http://dx.doi.org/10.1016/j.jbankfin.2013.01.017

[10] Shiryaev, A.N., Buhlmann, H., Delbaen, F. and Embrechts, P. (1995) No-Arbitrage, Change of Measure and Conditional Esscher Transformations. https://people.math.ethz.ch/ delbaen/ftp/preprints/BDES-CWI.pdf

[11] Shiryaev, A. (2003) Essentials of Stochastic Finance: Facts, Models and Theory. World Scientific, Singapore, 3.

[12] Oksendal, B. and Sulem, A. (2009) Applied Stochastic Control of Jump Diffusions. 3rd Edition, Springer, Berlin.

[13] Nkeki, C.I. (2013) Optimal Investment under Inflation Protection and Optimal Portfolios with Discounted Cash Flows Strategy. Journal of Mathematical Finance, 3, 130-137. http://dx.doi.org/10.4236/jmf.2013.31012

[14] Oksendal, B. (2010) Stochastic Differential Equations: An Introduction with Applications. 6th Edition, Springer, Berlin.

[15] Klebaner, F.C. (1998) Introduction to Stochastic Calculus with Applications. Imperial College Press, London. http://dx.doi.org/10.1142/p110

[16] Cont, R. and Tankov, P. (2004) Financial Modelling with Jump Processes. CRC Press, Boca Raton. 


\section{Appendix}

\section{Appendix A}

Assume that $f=e^{H_{t}}$ and $f \in \mathcal{C}^{2}$. Using Itô formula for sememartingales (see Jacod [?], Protter [?], Shiryaev [11], Shiryaev [10]) $f$, one obtains

$$
\begin{aligned}
f\left(H_{t}\right)= & f\left(H_{0}\right)+\int_{0}^{t} f^{\prime}\left(H_{s-}\right) \mathrm{d} H_{s}+\frac{1}{2} \int_{0}^{t} f^{\prime \prime}\left(H_{s-}\right) \mathrm{d}\left\langle H^{c}\right\rangle_{s} \\
& +\sum_{0<s \leq t}\left[f\left(H_{s}\right)-f\left(H_{s-}\right)-f^{\prime}\left(H_{s-}\right) \Delta H_{s}\right]
\end{aligned}
$$

to find our SDE, assume that $f(x)=e^{x}$ and substitute on Equation (47). Simplifying will give the following results

$$
\begin{aligned}
e_{t}^{H} & =e_{0}^{H}+\int_{0}^{t}\left(e^{H_{s-}}\right)^{\prime} \mathrm{d} H_{s}+\frac{1}{2} \int_{0}^{t}\left(e^{H_{s-}}\right)^{\prime \prime} \mathrm{d}\left\langle H^{c}\right\rangle_{s}+\sum_{0<s \leq t}\left[e^{H_{s}}-e^{H_{s-}}-\left(e^{H_{s-}}\right)^{\prime} \Delta H_{s}\right] \\
& =e_{0}^{H}+\int_{0}^{t} e^{H_{s-}} \mathrm{d} H_{s}+\frac{1}{2} \int_{0}^{t} e^{H_{s-}} \mathrm{d}\left\langle H^{c}\right\rangle_{s}+\sum_{0<s \leq t}\left[e^{H_{s}}-e^{H_{s-}}-e^{H_{s-}} \Delta H_{s}\right]
\end{aligned}
$$

Differentiating will give;

$$
\begin{aligned}
& \mathrm{d} e_{t}^{H}=0+\mathrm{d} \int_{0}^{t} e^{H_{s-}} \mathrm{d} H_{s}+\frac{1}{2} \mathrm{~d} \int_{0}^{t} e^{H_{s-}} \mathrm{d}\left\langle H^{c}\right\rangle_{s}+\mathrm{d} \sum_{0<s \leq t}\left[e^{H_{s}}-e^{H_{s-}}-e^{H_{s-}} \Delta H_{s}\right] \\
&=e^{H_{s-}} \mathrm{d} H_{s}+\frac{1}{2} e^{H_{s-}} \mathrm{d}\left\langle H^{c}\right\rangle_{s}+\left[e^{H_{s}}-e^{H_{s-}}-e^{H_{s-}} \Delta H_{s}\right] \\
&=e^{H_{s-}} \mathrm{d} H_{s}+\frac{1}{2} e^{H_{s-}} \mathrm{d}\left\langle H^{c}\right\rangle_{s}+\left(e^{\Delta H_{s}+H_{s-}}-e^{H_{s-}}-e^{H_{s-}} \Delta H_{s}\right), \Delta H_{s}=H_{s}-H_{s-} \\
&=e^{H_{s-}} \mathrm{d} H_{s}+\frac{1}{2} e^{H_{s-}} \mathrm{d}\left\langle H^{c}\right\rangle_{s}+e_{s-}^{H}\left(e^{\Delta H_{s}}-1-\Delta H_{s}\right) \\
&=e^{H_{t-}}\left\{\mathrm{d} H_{t}+\frac{1}{2} \mathrm{~d}\left\langle H^{c}\right\rangle_{t}+\left(e^{\Delta H_{s}}-1-\Delta H_{s}\right)\right\} \\
&=e^{H_{t-}}\left\{\mathrm{d} H_{t}+\frac{1}{2} \mathrm{~d}\left\langle H^{c}\right\rangle_{t}+\mathrm{d} \sum_{0<s \leq t}\left(e^{\Delta H_{s}}-1-\Delta H_{s}\right)\right\} \\
&=e^{H_{t-}} \mathrm{d}\left\{H_{t}+\frac{1}{2}\left\langle H^{c}\right\rangle_{t}+\sum_{0<s \leq t}\left(e^{\Delta H_{s}}-1-\Delta H_{s}\right)\right\} \\
& \quad \mathrm{d} e_{t}^{H}=e^{H_{t-}} \mathrm{d} \hat{H}_{t}
\end{aligned}
$$

Now the differential of the stock process is given by

$$
\mathrm{d} S_{t}=S_{t-} \mathrm{d} \hat{H}_{t}
$$

where

$$
\hat{H}_{t}=H_{t}+\frac{1}{2}\left\langle H^{c}\right\rangle_{s}+\sum_{0<s \leq t}\left(e^{\Delta H_{s}}-1-\Delta H_{s}\right)
$$

then, using Ito's formula for semimartingales (Protter [?]), we have

$$
\begin{aligned}
Y_{t} & =e^{X_{0}}+\int_{0}^{t}\left(e^{X_{s-}}\right)^{\prime} \mathrm{d} X_{s}+\frac{1}{2} \int_{0}^{t}\left(e^{X_{s-}}\right)^{\prime \prime} \mathrm{d}\left\langle X^{c}\right\rangle_{s}+\sum_{0 \leq s \leq t}\left(e^{X_{s}}-e^{X_{s-}}-\left(e^{X_{s-}}\right)^{\prime} \Delta X_{s}\right) \\
& =e^{X_{0}}+\int_{0}^{t} e^{X_{s-}} \mathrm{d} X_{s}+\frac{1}{2} \int_{0}^{t} e^{X_{s-}} \mathrm{d}\left\langle X^{c}\right\rangle_{s}+\sum_{0 \leq s \leq t}\left(e^{X_{s}}-e^{X_{s-}}-e^{X_{s-}} \Delta X_{s}\right)
\end{aligned}
$$


and in differential form, this can be expressed as

$$
\begin{aligned}
\mathrm{d} Y_{t} & =e^{X_{t-}} \mathrm{d} X_{t}+\frac{1}{2} e^{X_{t-}} \mathrm{d}\left\langle X^{c}\right\rangle_{t}+e^{X_{t}}-e^{X_{t-}}-e^{X_{t-}} \Delta X_{t}=e^{X_{t-}} \mathrm{d} X_{t}+\frac{1}{2} e^{X_{t-}} \mathrm{d}\left\langle X^{c}\right\rangle_{t}+e^{X_{t-}+\Delta X_{t}}-e^{X_{t-}}-e^{X_{t-}} \Delta X_{t} \\
& =e^{X_{t-}} \mathrm{d} X_{t}+\frac{1}{2} e^{X_{t-}} \mathrm{d}\left\langle X^{c}\right\rangle_{t}+e^{X_{t-}}\left(e^{\Delta X_{t}}-1-\Delta X_{t}\right) \\
& =e^{X_{t-}} \mathrm{d}\left[X_{t}+\frac{1}{2}\left\langle X^{c}\right\rangle_{t}+\sum_{0<s \leq t}\left(e^{\Delta X_{s}}-1-\Delta X_{s}\right)\right]=Y_{t-} \mathrm{d} \hat{X}_{t},
\end{aligned}
$$

\section{Appendix B}

Assuming $Y_{t}=\ln S_{t}$ and substituting it on the formula we get

$$
\begin{aligned}
& \mathrm{d} Y_{t}= \frac{\partial Y_{t}}{\partial t} \mathrm{~d} t+\frac{\partial Y_{t}}{\partial S_{t}}\left[\mathrm{~d} S_{t}\right]^{c}+\frac{1}{2} \frac{\partial^{2} Y_{t}}{\partial S_{t}^{2}}\left[\mathrm{~d} S_{t}^{c}\right]^{2}+\int_{|z| \leq 0}\left(\ln \left(S_{t}-S_{t} \varphi_{1}\right)-\ln S_{t}+\frac{\partial Y_{t}}{\partial S_{t}} S_{t} \varphi_{1}\right) v(\mathrm{~d} z) \\
&+\int_{|z| \leq 0}\left(\ln \left(S_{t}-S_{t} \varphi_{2}\right)-\ln S_{t}+\frac{\partial Y_{t}}{\partial S_{t}} S_{t} \varphi_{2}\right) v(\mathrm{~d} z)+\int_{\mathbb{R}}\left(\ln \left(S_{t}-S_{t} \varphi_{2}\right)-\ln S_{t}\right)(\mu-v)(\mathrm{d} z) \\
&= \alpha \mathrm{d} t+\sigma_{1} \mathrm{~d} W_{t}-\frac{1}{2} \sigma_{1}^{2} \mathrm{~d} t+\int_{|z| \leq 0}\left(\ln \left(1-\varphi_{1}\right)+\varphi_{1}\right) v(\mathrm{~d} z) \\
&+\int_{|z| \leq 0}\left(\ln \left(1-\varphi_{2}\right)+\varphi_{2}\right) v(\mathrm{~d} z)+\int_{\mathbb{R}}\left(\ln \left(1-\varphi_{2}\right)\right)(\mu-v)(\mathrm{d} z) \\
& \ln S_{t}-\ln S_{0}=\left(\alpha-\frac{1}{2} \sigma_{1}^{2}\right) t+\sigma_{1} W_{t}+\int_{0}^{t} \int_{|z| \leq 0}\left(\ln \left(1-\varphi_{1}\right)+\varphi_{1}\right) v(\mathrm{~d} z, \mathrm{~d} s) \\
& \quad+\int_{0}^{t} \int_{|z| \leq 0}\left(\ln \left(1-\varphi_{2}\right)+\varphi_{2}\right) v(\mathrm{~d} z, \mathrm{~d} s)+\int_{0}^{t} \int_{\mathbb{R}}\left(\ln \left(1-\varphi_{2}\right)\right)(\mu-v)(\mathrm{d} z, \mathrm{~d} s) \\
& S_{t}=S_{0} \exp \left\{\left(\alpha-\frac{1}{2} \sigma_{1}^{2}\right) t+\sigma_{1} W_{t}+\int_{0}^{t} \int_{|z| \leq 0}\left(\ln \left(1-\varphi_{1}\right)+\varphi_{1}\right) v(\mathrm{~d} z, \mathrm{~d} s)\right. \\
&\left.+\int_{0}^{t} \int_{|z| \leq 0}\left(\ln \left(1-\varphi_{2}\right)+\varphi_{2}\right) v(\mathrm{~d} z, \mathrm{~d} s)+\int_{0}^{t} \int_{\mathbb{R}}\left(\ln \left(1-\varphi_{2}\right)\right)(\mu-v)(\mathrm{d} z, \mathrm{~d} s)\right\}
\end{aligned}
$$

\section{Appendix C}

Let $f\left(t, P_{t}\right)=Y_{t}=\ln P(t)$ and

$$
\begin{aligned}
\mathrm{d} Y_{t}= & \frac{\partial Y}{\partial t} \mathrm{~d} t+\frac{\partial Y}{\partial P} \mathrm{~d} P^{c}+\frac{1}{2} \frac{\partial^{2} Y}{\partial P^{2}}[\mathrm{~d} P]^{2} \\
& +\int_{|z| \leq 1}\left(Y(t, P(t-)-\xi(t, z))-Y(t, P(t-))-\frac{\partial Y}{\partial P} \xi(t, z)\right) v(\mathrm{~d} t, \mathrm{~d} z) \\
= & \frac{1}{P}\left(P\left(-\phi \mathrm{d} W_{t}\right)\right)-\frac{1}{2} \frac{1}{P^{2}}\left[P\left(\phi \mathrm{d} W_{t}\right)\right]^{2} \\
& +\int_{|x| \leq 1}\left(\ln (P(t-)+P(t-) \psi(t, z))-\ln P(t-)-\frac{1}{P(t-)} P(t-) \psi(t, z)\right) v(\mathrm{~d} t, \mathrm{~d} z) \\
= & -\phi \mathrm{d} W_{t}-\frac{1}{2} \phi^{2} \mathrm{~d} t+\int_{|z| \leq 1}\left(\ln \frac{P(t-)(1+\psi(t, z))}{P(t-)}-\psi(t, z)\right) v(\mathrm{~d} t, \mathrm{~d} z) \\
= & -\phi \mathrm{d} W_{t}-\frac{1}{2} \phi^{2} \mathrm{~d} t+\int_{|z| \leq 1}(\ln (1+\psi(t, z))-\psi(t, z)) v(\mathrm{~d} t, \mathrm{~d} z)
\end{aligned}
$$




$$
\begin{gathered}
\mathrm{d} \ln P(t)=-\phi \mathrm{d} W_{t}-\frac{1}{2} \phi^{2} \mathrm{~d} t+\int_{|z| \leq 1}(\ln (1+\psi(t, z))-\psi(t, z)) v(\mathrm{~d} t, \mathrm{~d} z) \\
\ln P(t)-\ln P(0)=-\phi W_{t}-\frac{1}{2} \phi^{2} t+\int_{0}^{t} \int_{|z| \leq 1}(\ln (1+\psi(t, z))-\psi(t, z)) v(\mathrm{~d} z, \mathrm{~d} s) \\
\ln \frac{P(t)}{P(0)}=-\phi W_{t}-\frac{1}{2} \phi^{2} t+\int_{0}^{t} \int_{|z| \leq 1}(\ln (1+\psi(t, z))-\psi(t, z)) v(\mathrm{~d} z, \mathrm{~d} s) \\
\frac{P(t)}{P(0)}=\exp \left(-\phi W_{t}-\frac{1}{2} \phi^{2} t+\int_{0}^{t} \int_{|z| \leq 1}(\ln (1+\psi(t, z))-\psi(t, z)) v(\mathrm{~d} z, \mathrm{~d} s)\right) \\
P(t)=P(0) \exp \left(-\phi W_{t}-\frac{1}{2} \phi^{2} t+\int_{0}^{t} \int_{|z| \leq 1}(\ln (1+\psi(t, z))-\psi(t, z)) v(\mathrm{~d} z, \mathrm{~d} s)\right) \\
=P(0) \exp \left(-\phi W_{t}-\frac{1}{2} \phi^{2} t+\int_{0}^{t} \int_{|z| \leq 1} \psi_{1}(t, z) v(\mathrm{~d} z, \mathrm{~d} s)\right)
\end{gathered}
$$

\section{Appendix D}

$$
\begin{aligned}
\mathrm{d} X(t)= & X(t) \theta_{S}(t) \frac{\mathrm{d} S(t)}{S(t)}+X(t) \theta_{I}(t) \frac{\mathrm{d} B(t, I(t))}{B(t(I(t)))}+X(t) \theta_{0}(t) \frac{\mathrm{d} Q(t)}{Q(t)}+D(t) \mathrm{d} t \\
= & X(t) \theta_{S}(t) \frac{S_{t}}{S_{t}}\left(\alpha \mathrm{d} t+\sigma_{1} \cdot \mathrm{d} W(t)\right)+\frac{S_{t-}}{S_{t}}\left(\int_{|z| \leq 1} \varphi_{1}(\mu-v)(\mathrm{d} z, \mathrm{~d} s)+\int_{|z| \leq 1} \varphi_{2} v(\mathrm{~d} z, \mathrm{~d} s)\right) \\
& +X(t) \theta_{I}(t) \frac{\mathrm{d} B(t, I(t))}{B(t(I(t)))}+X(t) \theta_{0}(t) \frac{\mathrm{d} Q(t)}{Q(t)}+D(t) \mathrm{d} t \\
= & X(t) \theta_{S}(t)\left(\alpha \mathrm{d} t+\sigma_{1} \cdot \mathrm{d} W(t)\right)+\frac{S_{t-}}{S_{t}}\left(\int_{|z| \leq 1} \varphi_{1}(\mu-v)(\mathrm{d} z, \mathrm{~d} s)+\int_{|z| \leq 1} \varphi_{2} v(\mathrm{~d} z, \mathrm{~d} s)\right) \\
& +X(t) \theta_{I}(t)\left(\left(r+\sigma_{I} \phi_{I}\right) \mathrm{d} t+\sigma_{B} \mathrm{~d} W(t)\right)+X(t)\left(1-\theta_{s}(t)-\theta_{I}(t)\right)(r \mathrm{~d} t)+D(t) \mathrm{d} t \\
= & X(t) \theta_{S}(t)\left(\alpha \mathrm{d} t+\sigma_{1} \cdot \mathrm{d} W(t)\right)+\lambda\left(\int_{|z| \leq 1} \varphi_{1}(\mu-v)(\mathrm{d} z, \mathrm{~d} s)+\int_{|z| \leq 1} \varphi_{2} v(\mathrm{~d} z, \mathrm{~d} s)\right) \\
& +X(t) \theta_{I}(t)\left(\left(r+\sigma_{I} \phi_{I}\right) \mathrm{d} t+\sigma_{B} \mathrm{~d} W(t)\right)+X(t)\left(1-\theta_{s}(t)-\theta_{I}(t)\right)(r \mathrm{~d} t)+D(t) \mathrm{d} t \\
= & X(t) \theta_{S}(t)\left(\alpha \mathrm{d} t+\sigma_{1} \cdot \mathrm{d} W(t)\right)+X(t) \theta_{S}(t) \lambda\left(\int_{|z| \leq 1} \varphi_{1}(\mu-v)(\mathrm{d} z, \mathrm{~d} s)\right. \\
& \left.+\int_{|z| \leq 1} \varphi_{2}(v)(\mathrm{d} z, \mathrm{~d} s)\right)+X(t) \theta_{I}(t)\left(\left(r+\sigma_{I} \phi_{I}\right) \mathrm{d} t+\sigma_{B} \mathrm{~d} W(t)\right) \\
& +X(t)\left(1-\theta_{s}(t)-\theta_{I}(t)\right)(r \mathrm{~d} t)+D(t) \mathrm{d} t \\
= & X(t) \theta_{S}(\alpha-r) \mathrm{d} t+X(t) \theta_{I} \sigma_{I} \phi_{I} \mathrm{~d} t+r X(t) \mathrm{d} t+X(t) \theta S \sigma_{1} \mathrm{~d} W(t) \\
& +X(t) \theta_{I} \sigma B \mathrm{~d} W(t)+X(t) \theta_{S} \lambda\left(\int_{|z| \leq 1} \varphi_{1}(\mu-v)(\mathrm{d} z, \mathrm{~d} s)+\int_{|z| \leq 1} \varphi_{2} v(\mathrm{~d} z, \mathrm{~d} s)\right)+D(t) \mathrm{d} t \\
= & X(t)\left(\theta_{S}, \theta_{I}\right)^{\prime}\left(\begin{array}{l}
\alpha-r \\
\sigma_{I} \phi_{I}
\end{array}\right) \mathrm{d} t+r X(t) \mathrm{d} t+X(t)\left(\theta_{S}, \theta_{I}\right)^{\prime}\left(\begin{array}{l}
\sigma_{1} \\
\sigma_{B}
\end{array}\right) \mathrm{d} W(t) \\
& +X(t)\left(\theta_{S}, \theta_{I}\right)^{\prime}\left(\begin{array}{l}
\lambda \\
0
\end{array}\right)\left(\int_{|z| \leq 1} \varphi_{1}(\mu-v)(\mathrm{d} z, \mathrm{~d} s)+\int_{|z| \leq 1} \varphi_{2} v(\mathrm{~d} z, \mathrm{~d} s)\right)+D(t) \mathrm{d} t \\
& \left.X \Delta) \mathrm{d} t+(\Sigma \theta)^{\prime} \mathrm{d} W(t)+\theta \lambda\left(\int_{|z| \leq 1} \varphi_{1}(\mu-v)(\mathrm{d} z, \mathrm{~d} s)+\int_{|z| \leq 1} \varphi_{2} v(\mathrm{~d} z, \mathrm{~d} s)\right)\right\}+D(t) \mathrm{d} t \\
&
\end{aligned}
$$


then

$$
\begin{aligned}
\mathrm{d} X(t)= & \left(X_{t}(r+\theta \Delta)+D(t)\right) \mathrm{d} t+X_{t}(\Sigma \theta)^{\prime} \mathrm{d} W(t) \\
& +X_{t} \theta \lambda\left(\int_{|z| \leq 1} \varphi_{1}(\mu-v)(\mathrm{d} z, \mathrm{~d} s)+\int_{|z| \leq 1} \varphi_{2} v(\mathrm{~d} z, \mathrm{~d} s)\right)
\end{aligned}
$$

where

$$
\lambda=\exp \left(\alpha \delta t+\sigma_{1} \delta W_{t}+\int_{0}^{\delta t} \int_{|z| \leq 1} \varphi_{1}(\mu-v)(\mathrm{d} z, \mathrm{~d} s)+\int_{0}^{\delta t} \int_{|z| \leq 1} \varphi_{2} v(\mathrm{~d} z, \mathrm{~d} s)\right)
$$

with $\delta t=t-t-$ and $\delta W_{t}=W_{t}-W_{t-}$

$\lambda$ was found by simply dividing $S_{t}$ by $S_{t-}$ i.e.

$$
\begin{aligned}
\lambda & =\frac{S_{0} \exp \left(\alpha t+\sigma_{1} W_{t}+\int_{0}^{t} \int_{|z| \leq 1} \varphi_{1}(\mu-v)(\mathrm{d} z, \mathrm{~d} s)+\int_{0}^{t} \int_{|z| \leq 1} \varphi_{2}(v)(\mathrm{d} z, \mathrm{~d} s)\right)}{S_{0} \exp \left(\alpha t-+\sigma_{1} W_{t-}+\int_{0}^{t-} \int_{|z| \leq 1} \varphi_{1}(\mu-v)(\mathrm{d} z, \mathrm{~d} s)+\int_{0}^{t-} \int_{|z| \leq 1} \varphi_{2} v(\mathrm{~d} z, \mathrm{~d} s)\right)} \\
& =\exp \left(\alpha(t-t-)+\sigma_{1}\left(W_{t}-W_{t-}\right)+\int_{t-}^{t} \int_{|z| \leq 1} \varphi_{1}(\mu-v)(\mathrm{d} z, \mathrm{~d} s)+\int_{t-J_{|z| \leq 1}}^{t} \varphi_{2} v(\mathrm{~d} z, \mathrm{~d} s)\right) \\
& =\exp \left(\alpha \delta t+\sigma_{1} \delta W_{t}+\int_{0}^{\delta t} \int_{|z| \leq 1} \varphi_{1}(\mu-v)(\mathrm{d} z, \mathrm{~d} s)+\int_{0}^{\delta t} \int_{|z| \leq 1} \varphi_{2} v(\mathrm{~d} z, \mathrm{~d} s)\right)
\end{aligned}
$$

\section{Appendix E}

Let $f=C^{1,2}$ and define $Y(t)=f(t, V(t))=f(t, X(t), \Psi(t))$. Then $Y(t)$ is a stochastic process with jumps and

$$
\begin{aligned}
\mathrm{d} Y(t)= & \frac{\partial f}{\partial t}(t, X(t), \Psi(t)) \mathrm{d} t+\frac{\partial f}{\partial X(t)}(t, X(t), \Psi(t)) \mathrm{d} X^{c}(t) \\
& +\frac{\partial f}{\partial \Psi(t)}(t, X(t), \Psi(t)) \mathrm{d} \Psi^{c}(t) \\
& +\frac{1}{2} \frac{\partial^{2} f}{\partial X^{2}(t)}(t, X(t), \Psi(t))\left[\mathrm{d} X^{c}(t)\right]^{2} \\
& +\frac{1}{2} \frac{\partial^{2} f}{\partial \Psi^{2}(t)}(t, X(t), \Psi(t))\left[\mathrm{d} \Psi^{c}(t)\right]^{2} \\
& +\frac{\partial^{2} f}{\partial \Psi(t) \partial X(t)}(t, X(t), \Psi(t))\left[\mathrm{d} \Psi^{c}(t) \mathrm{d} X^{c}(t)\right] \\
& +\int\left\{f\left(t, V(t-)+\kappa_{1}(t, z, \omega)\right)-f(t, V(t-))-\frac{\partial f}{\partial X(t)}(t, V(t-)) \kappa_{1}(t, z, \omega)\right\} v(\mathrm{~d} z) \\
& +\int\left\{f\left(t, V(t-)+\kappa_{2}(t, z, \omega)\right)-f(t, V(t-))-\frac{\partial f}{\partial X(t)}(t, V(t-)) \kappa_{2}(t, z, \omega)\right\} v(\mathrm{~d} z) \\
& +\int\left\{f\left(t, V(t-)+\kappa_{2}(t, z, \omega)\right)-f(t, V(t-))\right\}(\mu-v)(\mathrm{d} z) \\
& +\int\left\{f\left(t, V(t-)+\kappa_{3}(t, z, \omega)\right)-f(t, V(t-))-\frac{\partial f}{\partial \Psi(t)}(t, V(t-)) \kappa_{3}(t, z, \omega)\right\} v(\mathrm{~d} z)
\end{aligned}
$$


take $Y=f(t, V(t))$ and substituting on 58 to have

$$
\begin{aligned}
\mathrm{d} Y(t)= & \frac{\partial f}{\partial t}(t, X(t), \Psi(t)) \mathrm{d} t+\frac{\partial f}{\partial X(t)}(t, X(t), \Psi(t))\left[X_{t}(r+\Delta \theta) \mathrm{d} t+\Sigma \theta \mathrm{d} W_{t}\right] \\
& +\frac{\partial f}{\partial \Psi(t)}(t, X(t), \Psi(t))\left[\Psi_{t}\left(r+\sigma_{D} \phi\right) \mathrm{d} t+\sigma_{D} \mathrm{~d} W_{t}\right] \\
& +\frac{1}{2} \frac{\partial^{2} f}{\partial X^{2}(t)}(t, X(t), \Psi(t))\left[X_{t}(r+\Delta \theta) \mathrm{d} t+\Sigma \theta \mathrm{d} W_{t}\right]^{2} \\
& +\frac{1}{2} \frac{\partial^{2} f}{\partial \Psi^{2}(t)}(t, X(t), \Psi(t))\left[(r+\Delta \theta) \mathrm{d} t+\Sigma \theta \mathrm{d} W_{t}\right]^{2} \\
& +\frac{\partial^{2} f}{\partial \Psi(t) \partial X(t)}(t, X(t), \Psi(t))\left[X_{t}(r+\Delta \theta) \mathrm{d} t+\Sigma \theta \mathrm{d} W_{t}\right]\left[\Psi_{t}\left(r+\sigma_{D} \phi\right) \mathrm{d} t+\sigma_{D} \mathrm{~d} W_{t}\right] \\
& +\int\left\{f\left(t, V(t-)+\kappa_{1}(t, z, \omega)\right)-f(t, V(t-))-\frac{\partial f}{\partial X(t)}(t, V(t-)) \kappa_{1}(t, z, \omega)\right\} v(\mathrm{~d} z) \\
& +\int\left\{f\left(t, V(t-)+\kappa_{2}(t, z, \omega)\right)-f(t, V(t-))-\frac{\partial f}{\partial X(t)}(t, V(t-)) \kappa_{2}(t, z, \omega)\right\} v(\mathrm{~d} z) \\
& +\int\left\{f\left(t, V(t-)+\kappa_{2}(t, z, \omega)\right)-f(t, V(t-))\right\}(\mu-v)(\mathrm{d} z) \\
& +\int\left\{f\left(t, V(t-)+\kappa_{3}(t, z, \omega)\right)-f(t, V(t-))-\frac{\partial f}{\partial \Psi(t)}(t, V(t-)) \kappa_{3}(t, z, \omega)\right\} v(\mathrm{~d} z)
\end{aligned}
$$$$
\mathrm{d} Y(t)=\frac{\partial f}{\partial t}(t, X(t), \Psi(t)) \mathrm{d} t+\frac{\partial f}{\partial X(t)}(t, X(t), \Psi(t))\left[X_{t}(r+\Delta \theta) \mathrm{d} t+\Sigma \theta \mathrm{d} W_{t}\right]
$$$$
+\frac{\partial f}{\partial \Psi(t)}(t, X(t), \Psi(t))\left[\Psi_{t}\left(r+\sigma_{D} \phi\right) \mathrm{d} t+\sigma_{D} \mathrm{~d} W_{t}\right]
$$$$
+\frac{1}{2} \frac{\partial^{2} f}{\partial X^{2}(t)}(t, X(t), \Psi(t))\left(X_{t}^{2} \Sigma^{2} \theta^{2} \mathrm{~d} t\right)
$$$$
+\frac{1}{2} \frac{\partial^{2} f}{\partial \Psi^{2}(t)}(t, X(t), \Psi(t))\left(\Psi_{t}^{2} \sigma_{D}^{\prime} \sigma_{D} \mathrm{~d} t\right)
$$$$
+\frac{\partial^{2} f}{\partial \Psi(t) \partial X(t)}(t, X(t), \Psi(t))\left(X_{t} \Psi_{t} \sigma_{D} \Sigma \theta \mathrm{d} t\right)
$$$$
+\int_{|z| \leq 1}\left\{f\left(t, V(t-)+\kappa_{1}(t, z, \omega)\right)-f(t, V(t-))-\frac{\partial f}{\partial X(t)}(t, V(t-)) \kappa_{1}(t, z, \omega)\right\} v(\mathrm{~d} z)
$$$$
+\int_{|z| \leq 1}\left\{f\left(t, V(t-)+\kappa_{2}(t, z, \omega)\right)-f(t, V(t-))-\frac{\partial f}{\partial X(t)}(t, V(t-)) \kappa_{2}(t, z, \omega)\right\} v(\mathrm{~d} z)
$$$$
+\int_{|z| \leq 1}\left\{f\left(t, V(t-)+\kappa_{2}(t, z, \omega)\right)-f(t, V(t-))\right\}(\mu-v)(\mathrm{d} z)
$$$$
+\int_{|z| \leq 1}\left\{f\left(t, V(t-)+\kappa_{3}(t, z, \omega)\right)-f(t, V(t-))-\frac{\partial f}{\partial \Psi(t)}(t, V(t-)) \kappa_{3}(t, z, \omega)\right\} v(\mathrm{~d} z)
$$

Choosing $f(t, V(t))=J(t, V(t))$ such that for a given portfolio strategy $\theta$ (not 
necessarily optimum, we introduce the associated utility

$$
J(t, x, \Psi, \theta)=\mathbb{E}_{t, x, \Psi}\left[U\left(V^{\theta}(T)\right)\right]
$$

Substituting $\kappa_{1}=x \theta \lambda \varphi_{1}, \quad \kappa_{2}=x \theta \lambda \varphi_{2}$ and $\kappa_{3}=\Psi \psi_{1}$ we now have

$$
\begin{aligned}
\mathrm{d} J(t, V(t))= & J_{t} \mathrm{~d} t+J_{x}\left[x(r+\Delta \theta) \mathrm{d} t+\Sigma \theta \mathrm{d} W_{t}\right] \\
& +J_{\Psi}\left[\Psi\left(r+\sigma_{D} \varphi\right) \mathrm{d} t+\sigma_{D} \mathrm{~d} W_{t}\right]+\frac{1}{2} J_{x x}\left(x^{2} \Sigma^{2} \theta^{2} \mathrm{~d} t\right) \\
& +\frac{1}{2} J_{\Psi \Psi}\left(\Psi^{2} \sigma_{D}^{\prime} \sigma_{D} \mathrm{~d} t\right)+J_{x \Psi}\left(x \Psi \sigma_{D} \Sigma \theta \mathrm{d} t\right) \\
& +\int_{|z| \leq 1}\left\{J\left(v+x \theta \lambda \varphi_{2}\right)-J(v)-J_{x} x \theta \lambda \varphi_{2}\right\} v(\mathrm{~d} z) \\
& +\int_{|z| \leq 1}\left\{J\left(v+x \theta \lambda \varphi_{1}\right)-J(v)-J_{x} x \theta \lambda \varphi_{1}\right\} v(\mathrm{~d} z) \\
& +\int_{|z| \leq 1}\left\{J\left(v+x \theta \lambda \varphi_{1}\right)-J(v)\right\}(\mu-v)(\mathrm{d} z) \\
& +\int_{|z| \leq 1}\left\{J\left(v+\Psi \psi_{1}\right)-J(v)-J_{\Psi} \Psi \psi_{1}\right\} v(\mathrm{~d} z) \\
= & \left(J_{t}+x(r+\Delta \theta) J_{x}+\Psi\left(r+\sigma_{D} \varphi\right) J_{\Psi}+\frac{1}{2} x^{2} \Sigma^{2} \theta^{2} J_{x x}\right. \\
& +\int_{|z| \leq 1}\left\{J\left(v+\Psi \psi_{1}\right)-J(v)-J_{\Psi} \Psi \psi_{1}\right\} v(\mathrm{~d} z) \\
& \left.+\frac{1}{2} \Psi \sigma^{2} \sigma_{D}^{2} J_{\Psi \Psi}+x \Psi \sigma_{D} \Sigma \theta J_{x \Psi}\right) \mathrm{d} t+\left(\Sigma \theta J_{x}+\sigma_{D} J_{\Psi}\right) \mathrm{d} W_{t} \\
& +\int_{|z| \leq 1}\left\{J\left(v+x \theta \lambda \varphi_{2}\right)-J(v)-J_{x} x \theta \lambda \varphi_{2}\right\} v(\mathrm{~d} z) \\
& +\int_{|z| \leq 1}\left\{J\left(v+x \theta \lambda \varphi_{1}\right)-J(v)-J_{x} x \theta \lambda \varphi_{1}\right\} v(\mathrm{~d} z) \\
& \\
& \\
& \\
&
\end{aligned}
$$

Integrating both sides we get

$$
\begin{aligned}
J(T, V(T))= & J(t, V(t))+\int_{t}^{T}\left(J_{s}+x(r+\Delta \theta) J_{x}+\Psi\left(r+\sigma_{D} \phi\right) J_{\Psi}\right. \\
& \left.+\frac{1}{2} x^{2} \Sigma^{2} \theta^{2} J_{x x}+\frac{1}{2} \Psi^{2} \sigma_{D}^{2} J_{\Psi \Psi}+x \Psi \sigma_{D} \Sigma \theta J_{x \Psi}\right) \mathrm{d} s \\
& +\int_{t}^{T}\left(x \Sigma \theta J_{x}+\sigma_{D} J_{\Psi}\right) \mathrm{d} W_{s} \\
& +\int_{t}^{T} \int_{|z| \leq 1}\left\{J\left(v+x \theta \lambda \varphi_{2}\right)-J(v)-J_{x} x \theta \lambda \varphi_{2}\right\} v(\mathrm{~d} z, \mathrm{~d} s) \\
& +\int_{t}^{T} \int_{|z| \leq 1}\left\{J\left(v+x \theta \lambda \varphi_{1}\right)-J(v)-J_{x} x \theta \lambda \varphi_{1}\right\} v(\mathrm{~d} z, \mathrm{~d} s) \\
& +\int_{t}^{T} \int_{|z| \leq 1}\left\{J\left(v+x \theta \lambda \varphi_{1}\right)-J(v)\right\}(\mu-v)(\mathrm{d} z, \mathrm{~d} s) \\
& +\int_{t}^{T} \int_{|z| \leq 1}\left\{J\left(v+\Psi \psi_{1}\right)-J(v)-J_{\Psi} \Psi \psi_{1}\right\} v(\mathrm{~d} z, \mathrm{~d} s)
\end{aligned}
$$

Taking the expectations on both sides we have 


$$
\begin{aligned}
& \mathbb{E}_{t, x, \Psi}[J(T, V(T))] \\
& =J(t, V(t))+\mathbb{E}_{t, x, \Psi}\left[\int _ { t } ^ { T } \left(J_{s}+x(r+\Delta \theta) J_{x}+\Psi\left(r+\sigma_{D} \phi\right) J_{\Psi}\right.\right. \\
& \left.+\frac{1}{2} x^{2} \Sigma^{2} \theta^{2} J_{x x}+\frac{1}{2} \Psi^{2} \sigma_{D}^{2} J_{\Psi \Psi}+x \Psi \sigma_{D} \Sigma \theta J_{x \Psi}\right) \mathrm{d} s \\
& +\int_{t}^{T} \int_{|z| \leq 1}\left\{J\left(v+x \theta \lambda\left(\varphi_{1}+\varphi_{2}\right)\right)-2 J(v)-J_{x} x \theta \lambda\left(\varphi_{1}+\varphi_{2}\right)\right\} v(\mathrm{~d} z, \mathrm{~d} s) \\
& \left.+\int_{t}^{T} \int_{|z| \leq 1}\left\{J\left(v+\Psi \psi_{1}\right)-J(v)-J_{\Psi} \Psi \psi_{1}\right\} v(\mathrm{~d} z, \mathrm{~d} s)\right]
\end{aligned}
$$

For simplicity we have

$$
\begin{aligned}
& \mathbb{E}_{t, x, \Psi}[J(T, V(T))] \\
& =J(t, x, \Psi)+\mathbb{E}_{t, x, \Psi}\left[\int _ { t } ^ { T } \left(J_{s}+x(r+\Delta \theta) J_{x}+\Psi\left(r+\sigma_{D} \phi\right) J_{\Psi}\right.\right. \\
& \left.+\frac{1}{2} x^{2} \Sigma^{2} \theta^{2} J_{x x}+\frac{1}{2} \Psi^{2} \sigma_{D}^{2} J_{\Psi \Psi}+x \Psi \sigma_{D} \Sigma \theta J_{x \Psi}\right) \mathrm{d} s \\
& +\int_{t}^{T} \int_{|z| \leq 1}\left\{J\left(v+x \theta \lambda \psi_{2}\right)-2 J(v)-J_{x} x \theta \lambda \psi_{2}\right\} v(\mathrm{~d} z, \mathrm{~d} s) \\
& \left.+\int_{t}^{T} \int_{|z| \leq 1}\left\{J\left(v+\Psi \psi_{1}\right)-J(v)-J_{\Psi} \Psi \psi_{1}\right\} v(\mathrm{~d} z, \mathrm{~d} s)\right]
\end{aligned}
$$

Where $\psi_{2}=\varphi_{1}+\varphi_{2}$ and $J(t, V(t))=J(t, x, \Psi)$. Since we know that $J\left(T, V^{\theta}(T)\right)=U\left(V^{\theta}(T)\right)$, we now have

$$
\begin{aligned}
& \mathbb{E}_{t, x, \Psi}\left[U\left(V^{\theta}(T)\right)\right] \\
& =J(t, x, \Psi)+\mathbb{E}_{t, x, \Psi}\left[\int _ { t } ^ { T } \left(J_{s}+x(r+\Delta \theta) J_{x}+\Psi\left(r+\sigma_{D} \phi\right) J_{\Psi}\right.\right. \\
& \left.+\frac{1}{2} x^{2} \Sigma^{2} \theta^{2} J_{x x}+\frac{1}{2} \Psi^{2} \sigma_{D}^{2} J_{\Psi \Psi}+x \Psi \sigma_{D} \Sigma \theta J_{x \Psi}\right) \mathrm{d} s \\
& +\int_{t}^{T} \int_{|z| \leq 1}\left\{J\left(v+x \theta \lambda \psi_{2}\right)-2 J(v)-J_{x} x \theta \lambda \psi_{2}\right\} v(\mathrm{~d} z, \mathrm{~d} s) \\
& \left.+\int_{t}^{T} \int_{|z| \leq 1}\left\{J\left(v+\Psi \psi_{1}\right)-J(v)-J_{\Psi} \Psi \psi_{1}\right\} v(\mathrm{~d} z, \mathrm{~d} s)\right]
\end{aligned}
$$

Which gives us

$$
\begin{aligned}
J(t, x, \Psi)= & \mathbb{E}_{t, x, \Psi}\left[U\left(V^{\theta}(T)\right)\right]-\mathbb{E}_{t, x, \Psi}\left[\int _ { t } ^ { T } \left(J_{s}+x(r+\Delta \theta) J_{x}+\Psi\left(r+\sigma_{D} \phi\right) J_{\Psi}\right.\right. \\
& \left.+\frac{1}{2} x^{2} \Sigma^{2} \theta^{2} J_{x x}+\frac{1}{2} \Psi^{2} \sigma_{D}^{2} J_{\Psi \Psi}+x \Psi \sigma_{D} \Sigma \theta J_{x \Psi}\right) \mathrm{d} s \\
& +\int_{t}^{T} \int_{|z| \leq 1}\left\{J\left(v+x \theta \lambda \psi_{2}\right)-2 J(v)-J_{x} x \theta \lambda \psi_{2}\right\} v(\mathrm{~d} z, \mathrm{~d} s) \\
& \left.+\int_{t}^{T} \int_{|z| \leq 1}\left\{J\left(v+\Psi \psi_{1}\right)-J(v)-J_{\Psi} \Psi \psi_{1}\right\} v(\mathrm{~d} z, \mathrm{~d} s)\right]
\end{aligned}
$$

By Equation (57), we have the integral on the right hand side being equals to zero. That is 


$$
\begin{aligned}
& \mathbb{E}_{t, x, \Psi}\left[\int _ { t } ^ { T } \left(J_{s}+x(r+\Delta \theta) J_{x}+\Psi\left(r+\sigma_{D} \phi\right) J_{\Psi}\right.\right. \\
& \left.+\frac{1}{2} x^{2} \Sigma^{2} \theta^{2} J_{x x}+\frac{1}{2} \Psi^{2} \sigma_{D}^{2} J_{\Psi \Psi}+x \Psi \sigma_{D} \Sigma \theta J_{x \Psi}\right) \mathrm{d} s \\
& +\int_{t}^{T} \int_{|z| \leq 1}\left\{J\left(v+x \theta \lambda \psi_{2}\right)-2 J(v)-J_{x} x \theta \lambda \psi_{2}\right\} v(\mathrm{~d} z, \mathrm{~d} s) \\
& \left.+\int_{t}^{T} \int_{|z| \leq 1}\left\{J\left(v+\Psi \psi_{1}\right)-J(v)-J_{\Psi} \Psi \psi_{1}\right\} v(\mathrm{~d} z, \mathrm{~d} s)\right]=0
\end{aligned}
$$

Differentiating both sides we obtain following partial differential equation with jumps.

$$
\begin{aligned}
& J_{t}+x(r+\Delta \theta) J_{x}+\Psi\left(r+\sigma_{D} \phi\right) J_{\Psi} \\
& +\frac{1}{2} x^{2} \Sigma^{2} \theta^{2} J_{x x}+\frac{1}{2} \Psi^{2} \sigma_{D}^{2} J_{\Psi \Psi}+x \Psi \sigma_{D} \Sigma \theta J_{x \Psi} \\
& +\int_{|z| \leq 1}\left\{J\left(v+x \theta \lambda \psi_{2}\right)-2 J(v)-J_{x} x \theta \lambda \psi_{2}\right\} v(\mathrm{~d} z) \\
& +\int_{|z| \leq 1}\left\{J\left(v+\Psi \psi_{1}\right)-J(v)-J_{\Psi} \Psi \psi_{1}\right\} v(\mathrm{~d} z)=0
\end{aligned}
$$

Consider the value function

$$
V(t, x, \Psi)=\sup _{\theta} J(t, x, \Psi, \theta)
$$

where $J$ is as in Equation (57) Under technical conditions, the value function $V$ satisfies

$$
\begin{aligned}
& U_{t}+\sup _{\theta}\left[\frac{1}{2} x^{2} \Sigma^{2} \theta^{2} U_{x x}+\Delta \theta U_{x}+x \Psi \sigma_{D} \Sigma \theta U_{x \Psi}\right. \\
& \left.+\int_{|z| \leq 1}\left\{U\left(v+x \theta \lambda \psi_{2}\right)-U_{x} x \theta \lambda \psi_{2}\right\} v(\mathrm{~d} z)\right]+\frac{1}{2} \Psi^{2} \sigma_{D}^{2} U_{\Psi \Psi} \\
& +\Psi\left(r+\sigma_{D} \phi\right) U_{\Psi}+r x U_{x} \int_{|z| \leq 1}\left\{U\left(v+\Psi \psi_{1}\right)-3 J(v)-U_{\Psi} \Psi \psi_{1}\right\} v(\mathrm{~d} z)=0
\end{aligned}
$$

This takes us to the HJB equation;

$$
U_{t}(t, v)+\max _{\theta \in \pi} \mathcal{L}^{\theta} \Phi(t, x, \Psi)=0
$$

where $\mathcal{L}^{\theta}$ is the second linear operator for jump diffusion. Hence

$$
\begin{aligned}
\mathcal{L}^{\theta} U= & x(r+\Delta \theta) U_{x}+\Psi\left(r+\sigma_{D} \phi\right) U_{\Psi}+\frac{1}{2} x^{2} \Sigma^{2} \theta^{2} U_{x x} \\
& +\frac{1}{2} \Psi^{2} \sigma_{D}^{2} U_{\Psi \Psi}+x \Psi \sigma_{D} \Sigma \theta U_{x \Psi} \\
& +\int_{|z| \leq 1}\left\{U\left(v+x \theta \lambda \psi_{2}\right)-2 U(v)-U_{x} x \theta \lambda \psi_{2}\right\} v(\mathrm{~d} z) \\
& +\int_{|z| \leq 1}\left\{U\left(v+\Psi \psi_{1}\right)-U(v)-U_{\Psi} \Psi \psi_{1}\right\} v(\mathrm{~d} z)
\end{aligned}
$$

Taking our utility function as

$$
U(v)=\frac{v^{1-\gamma}}{1-\gamma}
$$

We consider the function of $\theta$ which is 


$$
\begin{aligned}
\Gamma(\theta)= & \frac{1}{2} x^{2} \Sigma^{2} \theta^{2} U_{x x}+\Delta \theta U_{x}+x \Psi \sigma_{D} \Sigma \theta U_{x \Psi} \\
& +\int_{|z| \leq 1}\left\{U\left(v+x \theta \lambda \psi_{2}\right)-U_{x} x \theta \lambda \psi_{2}\right\} v(\mathrm{~d} z)
\end{aligned}
$$

Differentiating $U(v)$ and substitute on (63), we get

$$
\begin{aligned}
\Gamma(\theta)= & -\frac{1}{2} x^{2} \gamma \Sigma^{2} \theta^{2} v^{-\gamma-1}+\Delta \theta v^{-\gamma}-\gamma x \Psi \sigma_{D} \Sigma \theta v^{-\gamma-1} \\
& +\int_{|z| \leq 1}\left\{\frac{1}{1-\gamma}\left(v+x \theta \lambda \psi_{2}\right)^{1-\gamma}-v^{-\gamma} x \theta \lambda \psi_{2}\right\} v(\mathrm{~d} z)
\end{aligned}
$$

Since $\Gamma(\theta)$ is a concave function of $\theta$, to find its maximum we differentiate (64) with respect to $\theta$ to obtain

$$
\begin{aligned}
\frac{\partial}{\partial \theta} \Gamma(\theta)= & -x^{2} \gamma \Sigma^{2} \theta v^{-\gamma-1}+\Delta v^{-\gamma}-\gamma x \Psi \sigma_{D} \Sigma v^{-\gamma-1} \\
& +\int_{|z| \leq 1}\left\{x \lambda \psi_{2}\left(v+x \theta \lambda \psi_{2}\right)^{-\gamma}-v^{-\gamma} x \lambda \psi_{2}\right\} v(\mathrm{~d} z)
\end{aligned}
$$

For $v=0$ we can solve for $\theta$ because we have a linear equation below

$$
\begin{aligned}
x \Delta v^{-\gamma}-\gamma x^{2} \Sigma^{2} \theta v^{-\gamma-1}-\gamma x \Psi \sigma_{D} \Sigma v^{-\gamma-1}=0 \\
\theta=\frac{x \Delta v^{-\gamma}}{\gamma x^{2} \Sigma^{2} v^{-\gamma-1}}-\frac{\gamma x \Psi \sigma_{D} \Sigma v^{-\gamma-1}}{\gamma x^{2} \Sigma^{2} v^{-\gamma-1}} \\
=\frac{\Delta v}{\gamma x \Sigma^{2}}-\frac{\Psi \sigma_{D}}{x \Sigma}=\frac{\Sigma^{-2} \Delta v}{\gamma x}-\frac{\Psi \Sigma^{-1} \sigma_{D}}{x}
\end{aligned}
$$

and $\theta^{*}$ will be given by

$$
\theta^{*}=\frac{\Sigma^{-2} \Delta V^{*}(t)}{\gamma X^{*}(t)}-\frac{\Psi \Sigma^{-1} \sigma_{D}}{X^{*}(t)}
$$

substituting $\Sigma, \Delta$ and $\sigma^{D}$ as defined, we obtain the following

$$
\begin{aligned}
& \theta^{*}=\left(\begin{array}{c}
\frac{\phi_{I} V^{*}(t)}{\gamma \sigma_{I}^{2} X^{*}(t)}-\frac{\sigma_{1}^{D} \Psi(t)}{\sigma_{I} X^{*}(t)} \\
\frac{\sigma_{I}(\alpha-r)+\sigma_{S} \sigma_{I} \rho\left(\sigma_{S} \sqrt{1-\rho^{2}}-\sigma_{I}\right) V^{*}(t)}{\gamma \sigma_{S}^{2} \sigma_{I}\left(1-\rho^{2}\right) X^{*}(t)}-\frac{\left(\sigma_{I} \sigma_{2}^{D}-\rho \sigma_{S} \sigma_{1}^{D}\right) \Psi(t)}{\sigma_{S} \sigma_{I} \sqrt{1-\rho^{2}} X^{*}(t)}
\end{array}\right) \\
& \left(\begin{array}{c}
\theta_{I}^{*} \\
\theta_{S}^{*}
\end{array}\right)=\left(\begin{array}{c}
\frac{\phi_{I} V^{*}(t)}{\sigma_{I} \gamma X^{*}(t)}-\frac{\sigma_{1}^{D} \Psi(t)}{\sigma_{I} X^{*}(t)} \\
\frac{\left(\sigma_{I}(\alpha-r)+\sigma_{s} \phi_{I} \rho\left(\sigma_{s} \sqrt{1-\rho^{2}}-\sigma_{I}\right)\right) V^{*}(t)}{\sigma_{s}^{2} \sigma_{I}\left(1-\rho^{2}\right) \gamma X^{*}(t)}-\frac{\left(\sigma_{I} \sigma_{2}^{D}-\rho \sigma_{s} \sigma_{1}^{D}\right) \Psi(t)}{\sigma_{s} \sigma_{I} \sqrt{1-\rho^{2}} X^{*}(t)}
\end{array}\right)
\end{aligned}
$$

where

$$
\theta_{I}^{*}=\frac{\phi_{I} V^{*}(t)}{\sigma_{I} \gamma X^{*}(t)}-\frac{\sigma_{1}^{D} \Psi(t)}{\sigma_{I} X^{*}(t)}
$$

and 


$$
\theta_{S}^{*}=\frac{\left(\sigma_{I}(\alpha-r)+\sigma_{s} \phi_{I} \rho\left(\sigma_{s} \sqrt{1-\rho^{2}}-\sigma_{I}\right)\right) V^{*}(t)}{\sigma_{s}^{2} \sigma_{I}\left(1-\rho^{2}\right) \gamma X^{*}(t)}-\frac{\left(\sigma_{I} \sigma_{2}^{D}-\sigma_{s} \sigma_{1}^{D}\right) \Psi(t)}{\sigma_{s} \sigma_{I} \sqrt{1-\rho^{2}} X^{*}(t)}
$$

We can now see that

$$
\begin{aligned}
\theta_{0}^{*}= & 1-\theta_{I}^{*}-\theta_{s}^{*} \\
= & 1-\frac{\phi_{I} V^{*}(t)}{\sigma_{I} \gamma X^{*}(t)}+\frac{\sigma_{1}^{D} \Psi(t)}{\sigma_{I} X^{*}(t)}-\frac{\left(\sigma_{I}(\alpha-r)+\sigma_{s} \phi_{I} \rho\left(\sigma_{s} \sqrt{1-\rho^{2}}-\sigma_{I}\right)\right) V^{*}(t)}{\sigma_{s}^{2} \sigma_{I}\left(1-\rho^{2}\right) \gamma X^{*}(t)} \\
& +\frac{\left(\sigma_{I} \sigma_{2}^{D}-\sigma_{s} \sigma_{1}^{D}\right) \Psi(t)}{\sigma_{s} \sigma_{I} \sqrt{1-\rho^{2}} X^{*}(t)}
\end{aligned}
$$

Submit or recommend next manuscript to SCIRP and we will provide best service for you:

Accepting pre-submission inquiries through Email, Facebook, LinkedIn, Twitter, etc.

A wide selection of journals (inclusive of 9 subjects, more than 200 journals)

Providing 24-hour high-quality service

User-friendly online submission system

Fair and swift peer-review system

Efficient typesetting and proofreading procedure

Display of the result of downloads and visits, as well as the number of cited articles

Maximum dissemination of your research work

Submit your manuscript at: http://papersubmission.scirp.org/

Or contact jmf@scirp.org 